\title{
CONJUGATE DUALITY
}

\section{IN STOCHASTIC CONTROLS WITH DELAY}

\author{
ZIMENG WANG, ${ }^{*}$ University of Nottingham
}

DAVID J. HODGE, ${ }^{* *}$ University of Nottingham

HUILING LE, ${ }^{* * *}$ University of Nottingham

\begin{abstract}
This paper uses the method of conjugate duality to investigate a class of stochastic optimal control problems where state systems are described by stochastic differential equations with delay. For this, we first analyse a stochastic convex problem with delay and derive the expression for the corresponding dual problem. This enables us to obtain the relationship between the optimalities for the two problems. Then, by linking stochastic optimal control problems with delay with a particular type of stochastic convex problem, the result for the latter leads to sufficient maximum principles for the former.
\end{abstract}

Keywords: Anticipated backward stochastic differential equation; conjugate convex function; stochastic delay differential equation; stochastic maximum principle; stochastic optimal control with delay.

2010 Mathematics Subject Classification: Primary 93E20

Secondary 46A20; 60H07

\footnotetext{
* Postal address: School of Mathematical Sciences, University of Nottingham, University Park, NG7 2RD Nottingham, UK. Email address: pmxzw5@nottingham.ac.uk ** Postal address: School of Mathematical Sciences, University of Nottingham, University Park, NG7 2RD Nottingham, UK. Email address: david.hodge@nottingham.ac.uk *** Postal address: School of Mathematical Sciences, University of Nottingham, University Park, NG7 2RD Nottingham, UK. Email address: huiling.le@nottingham.ac.uk
} 


\section{Introduction}

There are many real-world problems providing applications for stochastic optimal control formulations. Examples include the quadratic loss minimization problem in portfolio optimization, and the consumption and investment problem in economics. It is well-known that Markovian optimal control problems can be solved by using either the method of dynamic programming or the stochastic maximum principle, the two methods having been developed separately and independently. In particular, the stochastic maximum principle typically involves a so-called Hamiltonian (function), a corresponding system of adjoint stochastic differential equations; the optimal control can be expressed in terms of the maximum of the Hamiltonian, analogous to deterministic cases which were originally studied by Pontryagin. We refer readers to [21, Chapter 3] for the general theory of the (Markovian) stochastic maximum principle.

Often, there is a need to extend these Markovian models to allow for time-lag or time delay effects. For example, see [8] for delayed models in estimating volatility of the price of a financial security. Also, although the efficient-market hypothesis states that current prices of assets reveal all the necessary information from the market, investors often take the historic performance of assets into consideration and use past information in modelling the wealth processes of portfolios. In such circumstances, if one uses stochastic delay differential equations (SDDEs) to model the state system, the corresponding portfolio optimization problem becomes a stochastic optimal control problem with (time) delay (see [3]). In contrast to Markovian optimal control problems, for control problems where the state systems are described by SDDEs, the backward equation of the value function, obtained by using the Bellman principle in the context of dynamic programming, depends on the initial path of the state process, and so it is generally infinite-dimensional. Note that, although recently developed functional Itô calculus (see [5] and [6]) may be applied to the delayed trajectory, the classical Itô formula cannot be applied to such a trajectory, Hence, it is generally difficult to obtain a corresponding finite-dimensional Hamilton-Jacobi-Bellman equation to solve the problem, except for some special cases. See for example [10] and [11].

Nevertheless, the Markovian stochastic maximum principle has been generalized to several stochastic control problems when state systems are described by various SDDEs, 
see [4], [12], [13], [14] and the references therein. The types of delay considered in these problems are usually either just discrete or both discrete and exponential moving average, noting that, if there is only an exponential moving average delay involved in a stochastic control problem, then it can be transferred to a higher-dimensional control problem with discrete delay. For example, for stochastic control problems with discrete delay, the authors of [4] and [12] establish sufficient maximum principles under different models and assumptions, where the associated adjoint equations are introduced and are described by anticipated backward stochastic differential equations (BSDEs) first studied by Peng and Yang in [15]. Sufficient maximum principles for stochastic control problems with both discrete and exponential moving average delays are obtained in [13] and [14]. However, the results in [13] and [14] are very different: in [13], the associated adjoint processes satisfy a triple of classical BSDEs with a restriction that one of them needs to be identically zero, while in [14] the associated adjoint process satisfies a single anticipated BSDE, but with a different Hamiltonian. As noted in [12], the restriction in [13] in effect reduces the control problem to a finite-dimensional one. Necessary maximum principles under various models are also studied by many authors; see, for example, [4], [12] and [14]. All these results are proved mainly by using results and techniques of stochastic calculus.

The conjugate duality method for analysing convex problems in the calculus of variations has played an important role in the study of classical optimal control problems. In the deterministic case, Rockafellar in [18] uses the concept of conjugate convex functions, described in his previous work [16], and the conjugate duality method to derive the corresponding dual problem and then obtains the conditions for optimality. After reformulating the control problem as a convex problem, Rockafellar obtains a sufficient maximum principle for the control problem, involving the Hamiltonian and associated adjoint equation. We refer readers to [17] and [19] for the method of conjugate duality and its applications in control theory. Bismut in [1] and [2] generalizes the work of [18] to Markovian convex and control problems. The method of conjugate duality has also been generalized to study deterministic convex problems with delay in [20], where the corresponding dual problem and the condition for optimality of the convex problems have been obtained in [20]. However, these results have not been 
connected to maximum principles for stochastic optimal controls with delay.

In this paper, departing from various stochastic calculus approaches used in studying stochastic control problems with delay in the literature, we generalize the results of [1] on Markovian control problems and of [20] on deterministic convex problems with delay. In particular, we extend the method of conjugate duality to study stochastic optimal control problems with either just discrete delay or both discrete and exponential moving average delays. For this and for clarity, we first investigate the stochastic convex problem with discrete delay: for given convex functions $L$ and $l$, minimize

$$
\Phi(X)=\mathrm{E}\left[\int_{0}^{T} L\left(t, X(t), X(t-\delta), \dot{X}(t), H_{X}(t)\right) d t\right]+\mathrm{E}[l(X(T))],
$$

where $X$ ranges through a certain family of Itô processes, $\dot{X}$ and $H_{X}$ denote respectively the drift and diffusion coefficients of $X$ and $\delta \in(0, T)$ is a given deterministic length of delay. We assume that $X(t)=x_{0}(t)$ for $t \in[-\delta, 0]$ for a given deterministic continuous function $x_{0}$. Note that, equivalently, we could maximize $\Phi$ if $L$ and $l$ were concave, for example, replacing $L$ and $l$ by $-L$ and $-l$. We investigate the corresponding dual problem and the conditions for optimization of the above problem. As noted in [20], the dependence on $X(t-\delta)$ in the convex problem results in its dependence on future values in its 'dual' process. Unlike the deterministic case, the 'time' cannot be reversed in the stochastic case. The novelty in our approach to overcome this difficulty lies in the use of conditional expectations in the characterization of dual processes and the use of the martingale representation theorem to identify them as solutions to BSDEs. Then, we consider stochastic optimal control problems with just discrete delay. We connect stochastic control problems with delay with stochastic convex problems. This allows us to use the conditions for optimality of the convex problems to prove sufficient maximum principles for stochastic control problems with delay. In particular, we derive the Hamiltonian and the associated adjoint equations and express the sufficient maximum principles in terms of them, where the adjoint equations are anticipated BSDEs. Finally, with fairly straightforward modifications, we extend our results in both the stochastic convex and control problems to allow the model to include both discrete and exponential moving average delays. Although it is not included in the paper, the approach that we take can easily be extended further to include a Lévy jump measure or regime-switching in stochastic convex problems 
with delay. This can then be used to obtain stochastic maximum principles in the corresponding control problems.

To be able to use the results in stochastic convex problems with delay, we require some extra conditions on the functions involved. Some of these conditions are stronger than those obtained using the stochastic calculus approach in the literature. Apart from these technical conditions, if only a discrete delay is involved, our result on the sufficient maximum principle is similar to those in [4] and [12] when their models are restricted to ours. Note that some apparent differences in the signs of some functions involved are the consequence of our problem being minimization and those in these papers being maximization. However, if both types of delay are involved, our result improves those in [14] and in [13], when the model in the latter is jump-free. Moreover, our approach of using the conjugate duality method unifies the Hamiltonian and the associated adjoint equations involved in the maximum principles for control problems with either just discrete delay or with both discrete and exponential moving average delays: those for the former are a special case for the latter.

The remainder of the paper is organized as follows. In Section 2, we describe the setting for the stochastic convex problem with (discrete) delay. In Section 3, we use conditional expectations to characterize dual processes and the martingale representation theorem to link them with the solutions of BSDEs. This enables us to derive the corresponding dual problem and, using the method of conjugate duality, obtain conditions for optimality. In Section 4, we concentrate on stochastic optimal control problems with discrete delay. We show how they can be reformulated as the convex problems described in Section 2. Then, the application of the conditions for optimality obtained in Section 3 leads to sufficient maximum principles for the stochastic control problem with discrete delay. We also give an example to show how the results in the previous section can be used to obtain the optimal control. In Section 5 , by modifying our previous arguments, we extend our results to stochastic control problems with both discrete and exponential moving average delays. 


\section{A stochastic convex problem with discrete delay}

Let $(\Omega, \mathcal{F}, \mathrm{P})$ be a complete probability space and $T \in(0, \infty)$ be a fixed time horizon. For a fixed positive integer $m$, write $B(t)=B(\omega, t)$ for a standard $m$-dimensional Brownian motion and $\{\mathcal{F}(t)\}_{t \in[0, T]}$ for the filtration generated by $B$ such that the usual conditions hold (see [9, Definition 2.25]).

In addition to $m$, we also fix an integer $n>0$ and introduce the following four functional spaces, where we have suppressed $\omega$ for notational simplicity:

$\mathbb{L}^{2}\left(\mathcal{F}(T) ; \mathbb{R}^{n}\right)$ : the space of $\mathcal{F}(T)$-measurable, $\mathbb{R}^{n}$-valued random variables $X$ for which the norm

$$
\|X\|_{2}=\left\{\mathrm{E}\left[|X|^{2}\right]\right\}^{1 / 2}
$$

is finite.

$\mathbb{L}_{\mathcal{F}}^{2 \infty}\left([0, T] ; \mathbb{R}^{n}\right)$ : the space of $\mathcal{F}(t)$-progressively measurable, $\mathbb{R}^{n}$-valued stochastic processes $X$ for which the norm

$$
\|X\|_{2 \infty}=\left\{\mathrm{E}\left[\operatorname{esssup}_{0 \leqslant t \leqslant T}|X(t)|^{2}\right]\right\}^{1 / 2}
$$

is finite.

$\mathbb{L}_{\mathcal{F}}^{21}\left([0, T] ; \mathbb{R}^{n}\right)$ : the space of $\mathcal{F}(t)$-progressively measurable, $\mathbb{R}^{n}$-valued stochastic processes $X$ for which the norm

$$
\|X\|_{21}=\left\{\mathrm{E}\left[\left(\int_{0}^{T}|X(t)| d t\right)^{2}\right]\right\}^{1 / 2}
$$

is finite.

$\mathbb{L}_{\mathcal{F}}^{22}\left([0, T] ; \mathbb{R}^{n \times m}\right)$ : the space of $\mathcal{F}(t)$-progressively measurable, $\mathbb{R}^{n \times m}$-valued stochastic processes $H$ for which the norm

$$
\|H\|_{22}=\left\{\mathrm{E}\left[\int_{0}^{T}|H(t)|^{2} d t\right]\right\}^{1 / 2}
$$

is finite, where elements in $\mathbb{R}^{n \times m}$ are represented by $n \times m$ matrices and so $|H(t)|^{2}=$ $\langle H(t), H(t)\rangle=\operatorname{tr}\left(H(t)^{\top} H(t)\right)$.

In what follows, we simply write the above functional spaces as $\mathbb{L}^{2}, \mathbb{L}_{\mathcal{F}}^{2 \infty}, \mathbb{L}_{\mathcal{F}}^{21}$ and $\mathbb{L}_{\mathcal{F}}^{22}$ respectively and, as above, suppress $\omega$ in functions and stochastic processes for notational simplicity, unless it is necessary for clarity. 
Write $\mathbb{X}=\mathbb{L}_{\mathcal{F}}^{21} \times \mathbb{L}_{\mathcal{F}}^{22}$, let $\delta \in(0, T)$ be fixed and $x_{0} \in \mathbb{C}\left([-\delta, 0] ; \mathbb{R}^{n}\right)$ be a given initial deterministic continuous function. Note that

$$
\max _{-\delta \leqslant t \leqslant 0}\left|x_{0}(t)\right|^{2}<\infty .
$$

We identify $\left(\dot{X}, H_{X}\right) \in \mathbb{X}$ with the continuous $\mathcal{F}(t)$-adapted stochastic process $X$ : $\Omega \times[-\delta, T] \rightarrow \mathbb{R}^{n}$ defined by

$$
\begin{aligned}
& X(t)=X(\omega, t) \\
= & \left\{\begin{array}{lc}
x_{0}(t), & t \in[-\delta, 0], \\
x_{0}(0)+\int_{0}^{t} \dot{X}(s) d s+\int_{0}^{t} H_{X}(s) d B(s), & t \in[0, T] .
\end{array}\right.
\end{aligned}
$$

Here the representation of $X$ by $\left(\dot{X}, H_{X}\right) \in \mathbb{X}$ is unique up to indistinguishability (see [9, Definition 1.3]). Note that, since it is continuous, $X$ is $\mathcal{F}(t)$-progressively measurable. Moreover, we define the delayed stochastic process $X_{\delta}$ associated with $X$ by

$$
X_{\delta}(t)=X(t-\delta), \quad t \in[0, T] .
$$

Proposition 2.1. For $X$ defined by (1), we have that $X_{\delta} \in \mathbb{L}_{\mathcal{F}}^{2 \infty}$ and $X(T) \in \mathbb{L}^{2}$.

Proof. By Doob's Maximal Inequality (see [9, page 14]), the definition of $X$ implies that $X \in \mathbb{L}_{\mathcal{F}}^{2 \infty}$ when it is restricted to $[0, T]$. Then, by noting that

$$
\sup _{0 \leqslant t \leqslant T}\left|X_{\delta}(t)\right|^{2} \leqslant 2\left\{\max _{-\delta \leqslant t \leqslant 0}\left|x_{0}(t)\right|^{2}+\sup _{0 \leqslant t \leqslant T}|X(t)|^{2}\right\}
$$

and that $|X(T)|^{2} \leqslant \sup _{0 \leqslant t \leqslant T}|X(t)|^{2}$, the required results follow.

Although the domain for $X$ defined by (1) is $[-\delta, T]$ for fixed $\omega \in \Omega$, for simplicity, we shall in the following regard $X$ as being in $\mathbb{L}_{\mathcal{F}}^{2 \infty}$ as its path in $[-\delta, 0]$ is fixed.

Let $L: \Omega \times[0, T] \times \mathbb{R}^{n} \times \mathbb{R}^{n} \times \mathbb{R}^{n} \times \mathbb{R}^{n \times m} \rightarrow \mathbb{R} \cup\{\infty\}$ and $l: \Omega \times \mathbb{R}^{n} \rightarrow \mathbb{R} \cup\{\infty\}$ be two given functions. Define functions $I_{L}$ on $\mathbb{L}_{\mathcal{F}}^{2 \infty} \times \mathbb{L}_{\mathcal{F}}^{2 \infty} \times \mathbb{L}_{\mathcal{F}}^{21} \times \mathbb{L}_{\mathcal{F}}^{22}$ and $J_{l}$ on $\mathbb{L}^{2}$ respectively by

$$
I_{L}(X, Y, Z, H)=\mathrm{E}\left[\int_{0}^{T} L(t, X(t), Y(t), Z(t), H(t)) d t\right]
$$

and

$$
J_{l}(X)=\mathrm{E}[l(X)]
$$


To ensure that $L$ and $l$ are measurable, and that $I_{L}$ and $J_{l}$ are strictly greater than $-\infty$, not identically $\infty$ and are convex, as well as to be able to apply the conjugate duality method to $I_{L}$ and $J_{l}$, we make the following assumptions throughout this paper.

Assumption I. (a) $L$ and $l$ are not identically $\infty$; when they are finite, $L$ is a lower semi-continuous convex function on $\mathbb{R}^{n} \times \mathbb{R}^{n} \times \mathbb{R}^{n} \times \mathbb{R}^{n \times m}$, for any $(\omega, t) \in \Omega \times[0, T]$, and $l$ is a lower semi-continuous convex function on $\mathbb{R}^{n}$, for any $\omega \in \Omega$.

(b) $L$ is $\mathcal{F}^{*} \times \mathcal{B}\left(\mathbb{R}^{n}\right) \times \mathcal{B}\left(\mathbb{R}^{n}\right) \times \mathcal{B}\left(\mathbb{R}^{n}\right) \times \mathcal{B}\left(\mathbb{R}^{n \times m}\right)$-measurable and $l$ is $\mathcal{F} \times \mathcal{B}\left(\mathbb{R}^{n}\right)$ measurable, where $\mathcal{F}^{*}$ for the completion of $\mathcal{F} \times \mathcal{B}([0, T])$ with respect to $d \mathrm{P} \otimes d t$.

Note that, in the presence of $(a)$, the condition $(b)$ is equivalent to $L$ and $l$ being 'normal convex integrands', a concept introduced in [16] (See also [18, page 180]). Assumption I ensures that, for any $(X, Y, Z, H) \in \mathbb{L}_{\mathcal{F}}^{2 \infty} \times \mathbb{L}_{\mathcal{F}}^{2 \infty} \times \mathbb{L}_{\mathcal{F}}^{21} \times \mathbb{L}_{\mathcal{F}}^{22}$ and $X_{T} \in$ $\mathbb{L}^{2}, L(\omega, t, X(\omega, t), Y(\omega, t), Z(\omega, t), H(\omega, t))$ and $l\left(\omega, X_{T}(\omega)\right)$ are $\mathcal{F}^{*}$ - and $\mathcal{F}$-measurable respectively.

Assumption II. (i) There exist $(X, Y, Z, H) \in \mathbb{L}_{\mathcal{F}}^{21} \times \mathbb{L}_{\mathcal{F}}^{21} \times \mathbb{L}_{\mathcal{F}}^{2 \infty} \times \mathbb{L}_{\mathcal{F}}^{22}$ and a $\mathbb{R}$-valued $\mathcal{F}(t)$-progressively measurable stochastic process $\tau_{1}$ satisfying

$$
\mathrm{E}\left[\int_{0}^{T}\left|\tau_{1}(t)\right| d t\right]<\infty
$$

such that for any $(x, y, z) \in \mathbb{R}^{n \times 3}$ and $h \in \mathbb{R}^{n \times m}$

$$
L(t, x, y, z, h) \geqslant\langle(x, y, z, h),(X(t), Y(t), Z(t), H(t))\rangle-\tau_{1}(t), \quad d \mathrm{P} \otimes d t \text {-a.s. }
$$

(ii) There exist $X \in \mathbb{L}^{2}$ and a $\mathbb{R}$-valued $\mathcal{F}(T)$-measurable random variable $\vartheta_{1}$ satisfying $\mathrm{E}\left[\left|\vartheta_{1}\right|\right]<\infty$, such that for any $x \in \mathbb{R}^{n}$

$$
l(x) \geqslant\langle x, X\rangle-\vartheta_{1}, \quad d \mathrm{P} \text {-a.s. }
$$

Assumption III. (i) There exist $(X, Y, Z, H) \in \mathbb{L}_{\mathcal{F}}^{2 \infty} \times \mathbb{L}_{\mathcal{F}}^{2 \infty} \times \mathbb{L}_{\mathcal{F}}^{21} \times \mathbb{L}_{\mathcal{F}}^{22}$ and a $\mathbb{R}$-valued $\mathcal{F}(t)$-progressively measurable stochastic process $\tau_{2}$ satisfying

$$
\mathrm{E}\left[\int_{0}^{T}\left|\tau_{2}(t)\right| d t\right]<\infty
$$

such that

$$
L(t, X(t), Y(t), Z(t), H(t)) \leqslant \tau_{2}(t), \quad d \mathrm{P} \otimes d t \text {-a.s. }
$$


(ii) There exist $X \in \mathbb{L}^{2}$ and a $\mathbb{R}$-valued $\mathcal{F}(T)$-measurable random variable $\vartheta_{2}$ satisfying $\mathrm{E}\left[\left|\vartheta_{2}\right|\right]<\infty$, such that

$$
l(\omega, X) \leqslant \vartheta_{2}, \quad d \mathrm{P} \text {-a.s. }
$$

Proposition 2.2. Under Assumptions I \& II for $L$ and $l$, we have that $I_{L}>-\infty$, $J_{l}>-\infty$, that both $I_{L}$ and $J_{l}$ are not identically $\infty$ and that both $I_{L}$ and $J_{l}$ are convex.

The proof of Proposition 2.2 is essentially the same as the proof for the deterministic case given in [18, Proposition 1]. Hence we omit it here.

Now, for given $L, l, x_{0}, \delta$ and for $X$ defined by (1), we define a function $\Phi$ of $X$ in terms of $I_{L}$ and $J_{l}$ by

$$
\Phi(X)=I_{L}\left(X, X_{\delta}, \dot{X}, H_{X}\right)+J_{l}(X(T)) .
$$

It follows directly from Proposition 2.2 that $\Phi>-\infty$ and that $\Phi$ is convex.

For such a function $\Phi$ we define, in a similar fashion to delay-free convex problems, the stochastic convex problem with discrete delay as follows.

Definition 2.1. The stochastic convex problem with discrete delay associated with $L$ and $l$ is to find $\bar{X} \in \mathbb{X}$ realizing

$$
\inf _{X \in \mathbb{X}} \Phi(X)
$$

where $X$ is identified with $\left(\dot{X}, H_{X}\right)$ using (1). We refer to the function $\Phi$ and the problem (3) as the primal function and problem respectively. Any $X \in \mathbb{X}$ such that $\Phi(X)<\infty$ will be called a feasible solution of this primal problem. Moreover, any feasible solution $\bar{X}$ that achieves the infimum in (3) will be called an optimal solution to the primal problem.

Note that, if $\Phi$ is identically $\infty$, no $X \in \mathbb{X}$ will be regarded as an optimal solution. Note also that our setting-up and definition of the primal function and problem bear a similarity to those studied in [1]. However, the extra delayed variable $X_{\delta}$ introduced in the primal function and problem is a function of $X$, and so the methods and results in [1] cannot be applied directly to our problem. 
Moreover, similarly to that for the corresponding deterministic convex problem with delay studied in [20, page 172], we define a family of perturbed functions $F$ of $\Phi$ on $\mathbb{X}$, parameterized by $(\theta, \xi, \eta) \in \mathbb{L}^{2} \times \mathbb{L}_{\mathcal{F}}^{2 \infty} \times \mathbb{L}_{\mathcal{F}}^{2 \infty}$, by

$$
F_{\theta, \xi, \eta}(X)=I_{L}\left(X+\xi, X_{\delta}+\eta, \dot{X}, H_{X}\right)+J_{l}(X(T)-\theta)
$$

Compared with the perturbed functions used for the delay-free deterministic convex problem in [18, Section 7] and for the Markovian convex problem in [1, Definition III-1], the function $F$ here depends on an extra parameter $\eta$ to take account of the delayed variable $X_{\delta}$ in $I_{L}$.

Accordingly, a family of perturbed optimization problems parameterized by $(\theta, \xi, \eta)$ is to find $\bar{X} \in \mathbb{X}$ realizing

$$
\inf _{X \in \mathbb{X}} F_{\theta, \xi, \eta}(X)
$$

This results in the corresponding optimal value function $\phi$ on $\mathbb{L}^{2} \times \mathbb{L}_{\mathcal{F}}^{2 \infty} \times \mathbb{L}_{\mathcal{F}}^{2 \infty}$ defined by

$$
\phi(\theta, \xi, \eta)=\inf _{X \in \mathbb{X}} F_{\theta, \xi, \eta}(X) .
$$

In particular, the relationship between $F$ and $\Phi$ gives that

$$
\phi(0,0,0)=\inf _{X \in \mathbb{X}} F_{0,0,0}(X)=\inf _{X \in \mathbb{X}} \Phi(X) .
$$

Clearly, $F$ is a composition of $\Phi$ with a certain affine mapping. Thus, $F$ is greater than $-\infty$ and is a convex function of $X$, which implies the convexity of $\phi$.

Proposition 2.3. The optimal value function $\phi$ defined by (5) is a convex function on $\mathbb{L}^{2} \times \mathbb{L}_{\mathcal{F}}^{2 \infty} \times \mathbb{L}_{\mathcal{F}}^{2 \infty}$.

\section{The dual problem and conditions for optimality}

We now apply a duality approach of convex analysis to obtain the corresponding dual problem to the primal problem given by Definition 2.1 and to relate the optimality of (3) with minimizers of the corresponding dual problem.

\subsection{Pairings and conjugate convex functions}

The fundamental notion for applying the conjugate duality method is the concept of paired linear spaces, or simply paired spaces, associated with a particular duality 
pairing, or simply pairing, which is an $\mathbb{R}$-valued bilinear form defined on the paired spaces. Following the convention described in [19, page 13], when we say that two linear spaces are paired spaces, then a pairing has been specified and these two spaces are respectively equipped with compatible topologies (see [19]) with respect to that pairing.

Throughout this paper, we shall pair Euclidean space $\mathbb{R}^{n}$ with itself via the Euclidean inner product. To derive the dual problem to (3), we pair $\mathbb{L}^{2}$ with itself via the pairing defined by

$$
\ll X_{T}, X_{T}^{*} \gg=\mathrm{E}\left[\left\langle X_{T}, X_{T}^{*}\right\rangle\right]
$$

pair $\mathbb{L}_{\mathcal{F}}^{22}$ with itself via the pairing defined by

$$
\ll H, H^{*} \gg=\mathrm{E}\left[\int_{0}^{T}\left\langle H(t), H^{*}(t)\right\rangle d t\right] ;
$$

pair $\mathbb{L}_{\mathcal{F}}^{21}$ with $\mathbb{L}_{\mathcal{F}}^{2 \infty}$ via the pairing defined by

$$
\ll X, X^{*} \gg=\mathrm{E}\left[\int_{0}^{T}\left\langle X(t), X^{*}(t)\right\rangle d t\right] .
$$

Since $\Phi$ is defined in terms of the functions $L$ and $l$, to derive its dual we let, for any fixed $(\omega, t) \in \Omega \times[0, T], L^{*}$ and $l^{*}$ be the usual conjugate convex functions of $L$ and $l$ with respect to the pairing given by the Euclidean inner product. A similar argument to that for [18, Theorem 2] shows that, since $L$ and $l$ satisfy Assumptions I, II \& III, $L^{*}$ and $l^{*}$ also satisfy the corresponding Assumptions I, II \& III. Moreover, since all four spaces defined in Section 2 are decomposable (see [16, page 532]), by Proposition 2.2, the conjugate duality given by [16, Theorem 2] can be generalized directly to relate $I_{L^{*}}$ and $J_{l^{*}}$ to $I_{L}$ and $J_{l}$ as follows, where $I_{L^{*}}$ and $J_{l^{*}}$ are defined similarly to $I_{L}$ and $J_{l}$ respectively.

Proposition 3.1. Under Assumptions I, II \& III, $I_{L}$ and $I_{L^{*}}$ are the conjugate convex functions of each other with respect to the pairing, between the product spaces $\mathbb{L}_{\mathcal{F}}^{2 \infty} \times$ $\mathbb{L}_{\mathcal{F}}^{2 \infty} \times \mathbb{L}_{\mathcal{F}}^{21} \times \mathbb{L}_{\mathcal{F}}^{22}$ and $\mathbb{L}_{\mathcal{F}}^{21} \times \mathbb{L}_{\mathcal{F}}^{21} \times \mathbb{L}_{\mathcal{F}}^{2 \infty} \times \mathbb{L}_{\mathcal{F}}^{22}$, induced directly from (7) and (8). Similarly, $J_{l}$ and $J_{l^{*}}$ are the conjugate convex functions of each other with respect to the pairing (6). 
Noting that $\phi$ defined by (5) is convex by Proposition 2.3, the conjugate convex function $\phi^{*}$ of $\phi$, with respect to the pairing induced from (6) and (8) between $\mathbb{L}^{2} \times$ $\mathbb{L}_{\mathcal{F}}^{2 \infty} \times \mathbb{L}_{\mathcal{F}}^{2 \infty}$ and $\mathbb{L}^{2} \times \mathbb{L}_{\mathcal{F}}^{21} \times \mathbb{L}_{\mathcal{F}}^{21}$, is given by

$$
\begin{aligned}
& \phi^{*}\left(\theta^{*}, \xi^{*}, \eta^{*}\right) \\
= & \sup _{(\theta, \xi, \eta) \in \mathbb{L}^{2} \times \mathbb{L}_{\mathcal{F}}^{2 \infty} \times \mathbb{L}_{\mathcal{F}}^{2 \infty}}\left\{\ll(\theta, \xi, \eta),\left(\theta^{*}, \xi^{*}, \eta^{*}\right) \gg-\phi(\theta, \xi, \eta)\right\} .
\end{aligned}
$$

Then, any solution to the optimization problem

$$
\inf _{\left(\theta^{*}, \xi^{*}, \eta^{*}\right) \in \mathbb{L}^{2} \times \mathbb{L}_{\mathcal{F}}^{21} \times \mathbb{L}_{\mathcal{F}}^{21}} \phi^{*}\left(\theta^{*}, \xi^{*}, \eta^{*}\right)
$$

is related to the optimality of our primal problem $(3)$. To see this, setting $(\theta, \xi, \eta)=$ $(0,0,0)$ on the right-hand-side of $(9)$, we have

$$
\phi^{*}\left(\theta^{*}, \xi^{*}, \eta^{*}\right) \geqslant-\phi(0,0,0)=-\inf _{X \in \mathbb{X}_{1}} \Phi(X),
$$

for all $\left(\theta^{*}, \xi^{*}, \eta^{*}\right) \in \mathbb{L}^{2} \times \mathbb{L}_{\mathcal{F}}^{21} \times \mathbb{L}_{\mathcal{F}}^{21}$, which implies

$$
\inf _{\left(\theta^{*}, \xi^{*}, \eta^{*}\right) \in \mathbb{L}^{2} \times \mathbb{L}_{\mathcal{F}}^{21} \times \mathbb{L}_{\mathcal{F}}^{21}} \phi^{*}\left(\theta^{*}, \xi^{*}, \eta^{*}\right)+\inf _{X \in \mathbb{X}} \Phi(X) \geqslant 0 .
$$

In particular, if there exist $\left(\bar{\theta}^{*}, \bar{\xi}^{*}, \bar{\eta}^{*}\right) \in \mathbb{L}^{2} \times \mathbb{L}_{\mathcal{F}}^{21} \times \mathbb{L}_{\mathcal{F}}^{21}$ and $\bar{X} \in \mathbb{X}$ such that the equality in (11) holds, then

$$
0 \leqslant \phi^{*}\left(\bar{\theta}^{*}, \bar{\xi}^{*}, \bar{\eta}^{*}\right)+\Phi(X)=-\Phi(\bar{X})+\Phi(X), \quad \forall X \in \mathbb{X}
$$

that is, $\bar{X}$ is an optimal solution to the primal problem (3).

\subsection{The dual problem}

For the Markovian convex problem studied in [1], the corresponding $\phi^{*}$ has been expressed in terms of the corresponding $I_{L^{*}}$ and $J_{l^{*}}$ in a similar manner to that for the corresponding primal function in terms of $I_{L}$ and $J_{l}$. Unfortunately, the introduction of the extra parameter $\eta^{*}$ in (9) to pair with $\eta$ in (5), due to the delayed variable $X_{\delta}$, makes this no longer the case; a phenomenon clear from the deterministic convex problem with delay studied in [20].

To find an expression for $\phi^{*}$, we write $\mathbb{P}=\mathbb{L}^{2} \times \mathbb{L}_{\mathcal{F}}^{21}$ and, for $\left(P_{T}, \dot{P}\right) \in \mathbb{P}$, define the continuous $\mathcal{F}(t)$-adapted stochastic process $P$ by

$$
P(t)=\mathrm{E}\left[P_{T}-\int_{t}^{T} \dot{P}(s) d s \mid \mathcal{F}(t)\right], \quad t \in[0, T] .
$$


Clearly, $P(0)$ is a constant. By the martingale representation theorem, there exists a unique $H_{P} \in \mathbb{L}_{\mathcal{F}}^{22}$ such that, for $t \in[0, T]$,

$$
P(t)=P_{T}-\int_{t}^{T} \dot{P}(s) d s-\int_{t}^{T} H_{P}(s) d B(s), \quad d \mathrm{P} \text {-a.s. }
$$

as $\{\mathcal{F}(t)\}_{t \in[0, T]}$ is generated by $B$. Moreover, by Doob's Maximal Inequality, it follows from (13) that, if $\left(P_{T}, \dot{P}\right) \in \mathbb{P}$, then $P \in \mathbb{L}_{\mathcal{F}}^{2 \infty}$. As for $X \in \mathbb{X}$, we shall identify $P$ with $\left(P_{T}, \dot{P}\right) \in \mathbb{P}$ using (12). However, unlike $X$, the identification (12) is implicit and it results in the explicit identification of $P$ with $\left(P_{T}, \dot{P}, H_{P}\right) \in \mathbb{L}^{2} \times \mathbb{L}_{\mathcal{F}}^{21} \times \mathbb{L}_{\mathcal{F}}^{22}$ by (13). Moreover, this explicit identification of $P$ shows that $P$ is the solution of a stochastic differential equation with a terminal, rather than an initial, condition, i.e. $P$ is the solution to a BSDE. Note that the corresponding $P$ in the deterministic convex problem with delay studied in [20, Proposition 3.1], which follows an ordinary differential equation with a terminal condition, can be equivalently expressed as the solution of an ordinary differential equation with an fixed initial condition, in a similar manner to that for $X$ in the corresponding primal problem described in [20, page 167]. The identification of $P$ here described by a BSDE is not equivalent to the identification for $X$ given by (1). The process $P \in \mathbb{P}$ defined in such a way plays an important role in our derivation of the expression for $\phi^{*}$ as given in the following theorem, which generalizes the result [20, Proposition 3.1] for the deterministic convex problem with delay.

Theorem 3.1. Suppose that Assumptions I, II \& III hold. For any given $\left(\theta^{*}, \xi^{*}, \eta^{*}\right) \in$ $\mathbb{L}^{2} \times \mathbb{L}_{\mathcal{F}}^{21} \times \mathbb{L}_{\mathcal{F}}^{21}$, let $\left(P_{T}, \dot{P}, \dot{Q}\right) \in \mathbb{P} \times \mathbb{L}_{\mathcal{F}}^{21}$ be defined by

$$
\left\{\begin{array}{l}
P_{T}=\theta^{*} \\
\dot{P}(t)=\xi^{*}(t)+\mathrm{E}\left[\eta^{*}(t+\delta) I_{[0, T-\delta]}(t) \mid \mathcal{F}(t)\right] \\
\dot{Q}(t)=\eta^{*}(t)
\end{array}\right.
$$

where $I_{A}$ denotes the indicator function of set $A$, and identify $P$ by $(12)$ with $\left(P_{T}, \dot{P}\right) \in$ 
$\mathbb{P}$. Then, $\Psi: \mathbb{P} \times \mathbb{L}_{\mathcal{F}}^{21} \rightarrow \mathbb{R} \cup\{\infty\}$, defined by

$$
\begin{aligned}
\Psi(P, \dot{Q})= & I_{L^{*}}\left(\dot{P}-\mathrm{E}\left[\dot{Q}(\cdot+\delta) I_{[0, T-\delta]}(\cdot) \mid \mathcal{F}(\cdot)\right], \dot{Q}, P, H_{P}\right)+J_{l^{*}}\left(-P_{T}\right) \\
& +\mathrm{E}\left[\left\langle P_{T}, x_{0}(0)\right\rangle\right]-\mathrm{E}\left[\int_{0}^{\delta}\left\langle\dot{Q}(t), x_{0}(t-\delta)\right\rangle d t\right] \\
& -\mathrm{E}\left[\int_{0}^{T}\left\langle\dot{P}(t), x_{0}(0)\right\rangle d t\right]
\end{aligned}
$$

satisfies $\Psi(P, \dot{Q})=\phi^{*}\left(\theta^{*}, \xi^{*}, \eta^{*}\right)$, where $H_{P}$ is specified by (13).

Proof. First, by Jensen's Inequality and Fubini's Theorem, the fact that $\eta^{*}$ is in $\mathbb{L}_{\mathcal{F}}^{21}$ implies that $\mathrm{E}\left[\eta^{*}(\cdot+\delta) I_{[0, T-\delta]}(\cdot) \mid \mathcal{F}(\cdot)\right] \in \mathbb{L}_{\mathcal{F}}^{21}$, so that $\dot{P}$ defined by (14) is in $\mathbb{L}_{\mathcal{F}}^{21}$.

Using (5) and $F$ defined by (4), we can re-express $\phi^{*}$ given by (9) as

$$
\begin{aligned}
& \phi^{*}\left(\theta^{*}, \xi^{*}, \eta^{*}\right) \\
& =\sup _{\substack{\left(\dot{X}, H_{X}\right) \in \mathbb{X} \\
(\theta, \xi, \eta) \in \mathbb{L}^{2} \times \mathbb{L}_{\mathcal{F}}^{2 \infty} \times \mathbb{L}_{\mathcal{F}}^{2 \infty}}}\left\{\mathrm{E}\left[\int_{0}^{T}\left(\left\langle\xi(t), \xi^{*}(t)\right\rangle+\left\langle\eta(t), \eta^{*}(t)\right\rangle\right) d t\right]\right. \\
& +\mathrm{E}\left[\left\langle\theta, \theta^{*}\right\rangle\right]-I_{L}\left(X+\xi, X_{\delta}+\eta, \dot{X}, H_{X}\right) \\
& \left.\quad-J_{l}(X(T)-\theta)\right\} .
\end{aligned}
$$

Then, setting $\theta^{\prime}=X(T)-\theta, \xi^{\prime}=X+\xi$ and $\eta^{\prime}=X_{\delta}+\eta$, it follows from (16) that

$$
\begin{aligned}
& \phi^{*}\left(\theta^{*}, \xi^{*}, \eta^{*}\right) \\
&= \sup _{\theta^{\prime} \in \mathbb{L}^{2}}\left\{\mathrm{E}\left[\left\langle\theta^{\prime},-\theta^{*}\right\rangle\right]-J_{l}\left(\theta^{\prime}\right)\right\} \\
&+\sup _{\substack{\left(X, H_{X}\right) \in \mathbb{X} \\
\left(\xi^{\prime}, \eta^{\prime}\right) \in \mathbb{L}_{\mathcal{F}}^{2 \infty} \times \mathbb{L}_{\mathcal{F}}}}\left\{\mathrm{E}\left[\int_{0}^{T}\left\langle\left(\xi^{\prime}(t), \eta^{\prime}(t)\right),\left(\xi^{*}(t), \eta^{*}(t)\right)\right\rangle d t\right]\right. \\
&+ \mathrm{E}\left[\left\langle X(T), \theta^{*}\right\rangle\right]-I_{L}\left(\xi^{\prime}, \eta^{\prime}, \dot{X}, H_{X}\right) \\
&\left.-\mathrm{E}\left[\int_{0}^{T}\left(\left\langle X(t), \xi^{*}(t)\right\rangle+\left\langle X_{\delta}(t), \eta^{*}(t)\right\rangle\right) d t\right]\right\} .
\end{aligned}
$$

To simplify this, we use the relationship between $X$ and $X_{\delta}$ to re-express the final 
term on the right-hand-side of (17) as

$$
\begin{aligned}
& \mathrm{E}\left[\int_{0}^{T}\left(\left\langle X(t), \xi^{*}(t)\right\rangle+\left\langle X_{\delta}(t), \eta^{*}(t)\right\rangle\right) d t\right] \\
& =\mathrm{E}\left[\int_{0}^{T}\left\langle X(t), \xi^{*}(t)+\eta^{*}(t+\delta) I_{[0, T-\delta]}(t)\right\rangle d t\right] \\
& \quad+\mathrm{E}\left[\int_{0}^{T}\left\langle x_{0}(t-\delta) I_{[0, \delta]}(t), \eta^{*}(t)\right\rangle d t\right] \\
& =\mathrm{E}\left[\int_{0}^{T}\left\langle X(t), \xi^{*}(t)+\mathrm{E}\left[\eta^{*}(t+\delta) I_{[0, T-\delta]}(t) \mid \mathcal{F}(t)\right]\right\rangle d t\right] \\
& \quad+\mathrm{E}\left[\int_{0}^{\delta}\left\langle x_{0}(t-\delta), \eta^{*}(t)\right\rangle d t\right] .
\end{aligned}
$$

On the other hand, using the expression (13) for $P$ and applying the Itô formula to $\langle P(t), X(t)\rangle$, we get

$$
\begin{aligned}
& \mathrm{E}\left[\left\langle X(T), P_{T}\right\rangle\right]-\left\langle x_{0}(0), P(0)\right\rangle \\
= & \mathrm{E}\left[\int_{0}^{T}\langle\dot{X}(t), P(t)\rangle d t\right]+\mathrm{E}\left[\int_{0}^{T}\langle X(t), \dot{P}(t)\rangle d t\right] \\
& +\mathrm{E}\left[\int_{0}^{T}\left\langle H_{X}(t), H_{P}(t)\right\rangle d t\right],
\end{aligned}
$$

recalling that $P(0)$ is a constant. Similarly, by applying the Itô formula to $\left\langle P(t), x_{0}(0)\right\rangle$, we have

$$
\left\langle x_{0}(0), P(0)\right\rangle=-\mathrm{E}\left[\int_{0}^{T}\left\langle x_{0}(0), \dot{P}(t)\right\rangle d t\right]+\mathrm{E}\left[\left\langle x_{0}(0), P_{T}\right\rangle\right] .
$$

Then, replacing $P_{T}$ and $\dot{P}$ in (19) and in (20) by their definitions (14), these two equations lead to

$$
\begin{aligned}
& \mathrm{E}\left[\int_{0}^{T}\left\langle X(t), \xi^{*}(t)+\mathrm{E}\left[\dot{Q}(t+\delta) I_{[0, T-\delta]}(t) \mid \mathcal{F}(t)\right]\right\rangle d t\right] \\
= & \mathrm{E}\left[\left\langle X(T), \theta^{*}\right\rangle-\left\langle x_{0}(0), \theta^{*}\right\rangle\right] \\
- & \mathrm{E}\left[\int_{0}^{T}\left\langle\left(\dot{X}(t), H_{X}(t)\right),\left(P(t), H_{P}(t)\right)\right\rangle d t\right] \\
- & \mathrm{E}\left[\int_{0}^{T}\left(\left\langle x_{0}(0), \eta^{*}(t)+\mathrm{E}\left[\dot{Q}(t+\delta) I_{[0, T-\delta]}(t) \mid \mathcal{F}(t)\right]\right\rangle\right) d t\right],
\end{aligned}
$$


the left-hand-side of which is equal to the first term of the right-hand-side of the second equality in (18). Finally, we substitute (18) into (17), using (21) and Proposition 3.1, to obtain

$$
\begin{aligned}
& \quad \phi^{*}\left(\theta^{*}, \xi^{*}, \eta^{*}\right) \\
& =\sup _{\substack{\left(\dot{X}, H_{X}\right) \in \mathbb{L}_{\mathcal{F}}^{21} \times \mathbb{L}_{\mathcal{F}}^{22} \\
\left(\xi^{\prime}, \eta^{\prime}\right) \in \mathbb{L}_{\mathcal{F}}^{2 \infty} \times \mathbb{L}_{\mathcal{F}}^{2 \infty}}}\left\{\ll\left(\xi^{\prime}, \eta^{\prime}, \dot{X}, H_{X}\right),\left(\xi^{*}, \dot{Q}, P, H_{P}\right) \gg-I_{L}\left(\xi^{\prime}, \eta^{\prime}, \dot{X}, H_{X}\right)\right\} \\
& \quad+\sup _{\theta^{\prime} \in \mathbb{L}^{2}}\left\{\ll \theta^{\prime},-\theta^{*} \gg-J_{l}\left(\theta^{\prime}\right)\right\} \\
& \quad+\mathrm{E}\left[\left\langle x_{0}(0), \theta^{*}\right\rangle\right]-\mathrm{E}\left[\int_{0}^{\delta}\left\langle x_{0}(t-\delta), \dot{Q}(t)\right\rangle d t\right] \\
& \quad-\mathrm{E}\left[\int_{0}^{T}\left\langle x_{0}(0), \xi^{*}(t)+\mathrm{E}\left[\dot{Q}(t+\delta) I_{[0, T-\delta]}(t) \mid \mathcal{F}(t)\right]\right\rangle d t\right] \\
& =I_{L^{*}}\left(\dot{P}-\mathrm{E}\left[\dot{Q}(\cdot+\delta) I_{[0, T-\delta]}(\cdot) \mid \mathcal{F}(\cdot)\right], \dot{Q}, P, H_{P}\right)+J_{l^{*}}\left(-P_{T}\right) \\
& +\mathrm{E}\left[\left\langle P_{T}, x_{0}(0)\right\rangle\right]-\mathrm{E}\left[\int_{0}^{\delta}\left\langle\dot{Q}(t), x_{0}(t-\delta)\right\rangle d t\right]-\mathrm{E}\left[\int_{0}^{T}\left\langle\dot{P}(t), x_{0}(0)\right\rangle d t\right],
\end{aligned}
$$

as required.

Although the relationship we obtained between $\Psi$ and $\phi^{*}$ bears some similarity to that between the corresponding functions obtained [20] for the deterministic convex problem with delay, our proof is different from that in [20]. In particular, we need to deal with the issue of an anticipated (or time advanced) variable.

Using (14), we can re-express the pairing $\ll(\theta, \xi, \eta),\left(\theta^{*}, \xi^{*}, \eta^{*}\right) \gg$ in terms of $(P, \dot{Q})$ as

$$
\begin{aligned}
& \ll(\theta, \xi, \eta),(P, \dot{Q}) \gg \\
&=\ll(\theta, \xi, \eta),\left(P_{T}, \dot{P}-\mathrm{E}\left[\dot{Q}(\cdot+\delta) I_{[0, T-\delta]}(\cdot) \mid \mathcal{F}(\cdot)\right], \dot{Q}\right) \gg \\
&=\mathrm{E}\left[\int_{0}^{T}\left\langle\xi(t), \dot{P}(t)-\mathrm{E}\left[\dot{Q}(t+\delta) I_{[0, T-\delta]}(t) \mid \mathcal{F}(t)\right]\right\rangle d t\right] \\
& \quad+\mathrm{E}\left[\left\langle\theta, P_{T}\right\rangle\right]+\mathrm{E}\left[\int_{0}^{T}\langle\eta(t), \dot{Q}(t)\rangle d t\right],
\end{aligned}
$$

where $P$ is identified with $\left(P_{T}, \dot{P}\right)$ via (12). This generalizes the pairing for the deterministic convex problem with delay given in [20, page 183]. Then, using the 
pairing $(22)$ and Theorem 3.1, we can re-express $\Psi(P, \dot{Q})$ given by (15) as

$$
\Psi(P, \dot{Q})=\sup _{(\theta, \xi, \eta) \in \mathbb{L}^{2} \times \mathbb{L}_{\mathcal{F}}^{2 \infty} \times \mathbb{L}_{\mathcal{F}}^{2 \infty}}\{\ll(P, \dot{Q}),(\theta, \xi, \eta) \gg-\phi(\theta, \xi, \eta)\} .
$$

By using Proposition 3.1 and noting Proposition 2.2, we see that $\Psi$ is strictly greater than $-\infty$ and is convex.

Definition 3.1. $\Psi$ is called a stochastic convex dual function of $\Phi$. The corresponding stochastic convex dual problem to (3) over $\mathbb{P} \times \mathbb{L}_{\mathcal{F}}^{21}$ is to find $(\bar{P}, \dot{\bar{Q}}) \in \mathbb{P} \times \mathbb{L}_{\mathcal{F}}^{21}$ realizing

$$
\inf _{(P, \dot{Q}) \in \mathbb{P} \times \mathbb{L}_{\mathcal{F}}^{21}} \Psi(P, \dot{Q}) .
$$

Similarly to the primal problem defined by Definition 2.1 , any $(P, \dot{Q}) \in \mathbb{P} \times \mathbb{L}_{\mathcal{F}}^{21}$ such that $\Psi(P, \dot{Q})<\infty$ will be called a feasible solution of the dual problem. we shall call a feasible solution $(\bar{P}, \bar{Q})$ which achieves the infimum in (24) an optimal solution to the dual problem.

Unlike the classical convex problem, although we call $\Psi$ the dual to $\Phi$, the space $\mathbb{P} \times \mathbb{L}_{\mathcal{F}}^{21}$ on which $\Psi$ is defined is not the paired space, with respect to the pairing defined in Section 2, to the space $\mathbb{X}$ on which $\Phi$ is defined on account of the fact that the convex problems we study also depends $X_{\delta}$. The reason that $\Psi$ is called the dual to $\Phi$ will become clear in the next subsection.

If there is no delay in the model, corresponding to $\delta=0, X_{\delta}$ is identical with $X$ and so there exists a function $\hat{L}: \Omega \times[0, T] \times \mathbb{R}^{n} \times \mathbb{R}^{n} \times \mathbb{R}^{n \times m} \rightarrow \mathbb{R} \cup\{\infty\}$ satisfying the corresponding Assumptions I, II \& III such that $L(\omega, t, x, x, z, h)=\hat{L}(\omega, t, x, z, h)$. Then, the optimal value function $\phi$, corresponding to $\hat{L}$ and $l$, depends only on $(\theta, \xi)$. Hence, Theorem 3.1 gives that $P=\left(P_{T}, \dot{P}\right) \in \mathbb{P}$ is identical with $\left(\theta^{*}, \xi^{*}\right)$, so that $\Psi(P)=\phi^{*}\left(\theta^{*}, \xi^{*}\right)$, and

$$
\Psi(P)=I_{\hat{L}^{*}}\left(\dot{P}, P, H_{P}\right)+J_{l^{*}}\left(-P_{T}\right)+\mathrm{E}\left[\left\langle P_{T}, x_{0}(0)\right\rangle\right]-\mathrm{E}\left[\int_{0}^{T}\left\langle\dot{P}(t), x_{0}(0)\right\rangle d t\right] .
$$

Applying the same technique as that in (20) to the last two terms on the right-hand-side of the above equation, we obtain that

$$
\Psi(P)=I_{\hat{L}^{*}}\left(\dot{P}, P, H_{P}\right)+J_{l^{*}}\left(-P_{T}\right)+\left\langle P(0), x_{0}(0)\right\rangle,
$$

recovering the dual function given in [1, Definition II-1] with fixed initial value $P(0)$. 


\subsection{Relationship between the optimalities for dual problems}

The following relationship between the primal function $\Phi$ and its dual function $\Psi$ is a direct consequence of (10) and Theorem 3.1.

Proposition 3.2. For any $X=\left(\dot{X}, H_{X}\right) \in \mathbb{X}$ and $(P, \dot{Q}) \in \mathbb{P} \times \mathbb{L}_{\mathcal{F}}^{21}$,

$$
\Phi(X)+\Psi(P, \dot{Q}) \geqslant 0 .
$$

We now use stochastic calculus to obtain the relationships between the optimal solutions of the primal and its dual problems as follows. This result generalizes [1, Theorem IV-2] for the Markovian convex problems. In particular, the third equivalent condition given below provides the crucial basis in the next section for us to derive the Hamiltonian and the associated adjoint equation for stochastic optimal control problems with discrete delay.

Theorem 3.2. For any given $\bar{X} \in \mathbb{X}$ and $(\bar{P}, \dot{\bar{Q}}) \in \mathbb{P} \times \mathbb{L}_{\mathcal{F}}^{21}$, the following three statements are equivalent:

(i)

$$
\Phi(\bar{X})+\Psi(\bar{P}, \dot{\bar{Q}})=0 .
$$

(ii) $\bar{X}$ and $(\bar{P}, \dot{\bar{Q}})$ are respectively optimal solutions to the primal problem (3) and its dual problem (24), and the equality in (25) is attained.

(iii)

$$
\begin{aligned}
& L^{*}\left(t, \dot{\bar{P}}(t)-\mathrm{E}\left[\dot{\bar{Q}}(t+\delta) I_{[0, T-\delta]}(t) \mid \mathcal{F}(t)\right], \dot{\bar{Q}}(t), \bar{P}(t), H_{\bar{P}}(t)\right) \\
& +L\left(t, \bar{X}(t), \bar{X}_{\delta}(t), \dot{\bar{X}}(t), H_{\bar{X}}(t)\right)-\left\langle\dot{\bar{Q}}(t), \bar{X}_{\delta}(t)\right\rangle \\
& -\left\langle\dot{\bar{P}}(t)-\mathrm{E}\left[\dot{\bar{Q}}(t+\delta) I_{[0, T-\delta]}(t) \mid \mathcal{F}(t)\right], \bar{X}(t)\right\rangle \\
& -\left\langle\left(\bar{P}(t), H_{\bar{P}}(t)\right),\left(\dot{\bar{X}}(t), H_{\bar{X}}(t)\right)\right\rangle=0, \quad d \mathrm{P} \otimes d t \text {-a.s. }
\end{aligned}
$$

and

$$
l(\bar{X}(T))+l^{*}\left(-\bar{P}_{T}\right)+\left\langle\bar{P}_{T}, \bar{X}(T)\right\rangle=0, \quad d \mathrm{P} \text {-a.s. }
$$

where $H_{\bar{P}}$ is specified by $\bar{P}$ via (13).

Note that if $\partial L$ and $\partial l$ denote the sub-differential sets of $L$ and $l$, conditions (27) 
and (28) are respectively equivalent to

$$
\begin{aligned}
& \left(\dot{\bar{P}}(t)-\mathrm{E}\left[\dot{\bar{Q}}(t+\delta) I_{[0, T-\delta]}(t) \mid \mathcal{F}(t)\right], \dot{\bar{Q}}(t), \bar{P}(t), H_{\bar{P}}(t)\right) \\
\in & \partial L\left(t, \bar{X}(t), \bar{X}_{\delta}(t), \dot{\bar{X}}(t), H_{\bar{X}}(t)\right), \quad d \mathrm{P} \otimes d t \text {-a.s. }
\end{aligned}
$$

and

$$
-\bar{P}(T) \in \partial l(\bar{X}(T)), \quad d \mathrm{P} \text {-a.s. }
$$

(see [18, page 207]).

Proof. (i) $\Leftrightarrow(i i)$ : Suppose that (26) holds. Then, the equality in (25) is attained. Moreover, it follows from (25) that $(\bar{P}, \dot{\bar{Q}})$ is an optimal solution to (24) and that $\bar{X}$ is an optimal solution to (3).

Conversely, if $\bar{X}$ and $(\bar{P}, \dot{\bar{Q}})$ are optimal solutions to (3) and (24) respectively, then (26) follows by combining (25) with the assumption that the equality therein is attained.

$($ i) $\Leftrightarrow$ (iii): Suppose that $(27)$ and $(28)$ hold for the given $\bar{X}$ and $(\bar{P}, \dot{\bar{Q}})$. Taking the integral of the left-hand-side of (27) over $[0, T]$, adding the left-hand-side of (28) and then taking the expectation, we have (26) using the expressions (2) for $\Phi$ and (15) for $\Psi$.

Conversely, it follows from (2) and (15) that (26) is equivalent to

$$
\mathrm{E}\left[\int_{0}^{T} A_{1}(t) d t\right]+\mathrm{E}\left[A_{2}\right]=0
$$

where $A_{1}$ is the process defined by the left-hand-side of (27) and $A_{2}$ is the random variable defined by the left-hand-side of (28). Since, for fixed $(\omega, t) \in \Omega \times[0, T], L^{*}$ and $l^{*}$ are the conjugate convex functions of $L$ and $l$ respectively, $A_{1}$ and $A_{2}$ are nonnegative. Then, the equality (29) implies that $A_{1}(t)=0, d \mathrm{P} \otimes d t$-a.s., and $A_{2}=0$, $d$ P-a.s., so that both (27) and (28) hold.

\section{A stochastic optimal control problem with discrete delay}

Having obtained the conditions for optimality of the stochastic convex problem with delay, we now turn our attention to the stochastic optimal control problem with discrete delay. 
Let $\mathbb{U} \subset \mathbb{R}^{r}$ be a convex set, where $r>0$ is a given integer; $b:[0, T] \times \mathbb{R}^{n} \times \mathbb{R}^{n} \times \mathbb{U} \rightarrow$ $\mathbb{R}^{n}$ and $\sigma:[0, T] \times \mathbb{R}^{n} \times \mathbb{R}^{n} \times \mathbb{U} \rightarrow \mathbb{R}^{n \times m}$ be two given measurable functions and the continuous $\mathcal{F}(t)$-adapted state process $X: \Omega \times[-\delta, T] \rightarrow \mathbb{R}^{n}$ be described by the controlled SDDE

$$
\left\{\begin{aligned}
d X(t)= & b\left(t, X(t), X_{\delta}(t), u(t)\right) d t & & \\
& +\sigma\left(t, X(t), X_{\delta}(t), u(t)\right) d B(t), & & t \in[0, T], \\
X(t)= & x_{0}(t), & & t \in[-\delta, 0],
\end{aligned}\right.
$$

where $x_{0}, X_{\delta}, \delta$ are as defined in Section 2 and $u: \Omega \times[0, T] \rightarrow \mathbb{U}$ is an $\mathcal{F}(t)$-adapted control process. For given continuous functions $G:[0, T] \times \mathbb{R}^{n} \times \mathbb{R}^{n} \times \mathbb{U} \rightarrow \mathbb{R}$ and $g: \mathbb{R}^{n} \rightarrow \mathbb{R}$, the cost functional $J$ associated with the controlled SDDE (30) is defined by

$$
J(u)=\mathrm{E}\left[\int_{0}^{T} G\left(t, X(t), X_{\delta}(t), u(t)\right) d t+g(X(T))\right] .
$$

Let $\mathcal{U}$ denote the space of admissible controls $u$ for which the controlled SDDE (30) admits a unique strong solution $\{X(t)\}_{t \in[-\delta, T]}$ and the cost functional $J$ is finite.

Definition 4.1. The stochastic optimal control problem with discrete delay associated with the controlled $\operatorname{SDDE}(30)$ and the cost functional $J$ is to find $\bar{u} \in \mathcal{U}$ realizing

$$
\inf _{u \in \mathcal{U}} J(u)
$$

We shall call $\bar{u}$ an optimal control.

Note that, this optimal control problem is a special case of the stochastic optimal control problems considered in $[4,12]$, where the models also include the discrete delayed control $u_{\delta}$.

\subsection{Reformulation of the problem}

To use the results for the stochastic convex problem with delay, obtained in the previous section, to study the control problem (31), we link the problem (31) with a particular convex problem (3) as follows. For $(\omega, t, x, y, z, h) \in \Omega \times[0, T] \times \mathbb{R}^{n} \times \mathbb{R}^{n} \times$ $\mathbb{R}^{n} \times \mathbb{R}^{n \times m}$, define the set $\mathcal{C}=\mathcal{C}(t, x, y, z, h)$ by

$$
\mathcal{C}(t, x, y, z, h)=\{u \in \mathbb{U} \mid z=b(t, x, y, u) \text { and } h=\sigma(t, x, y, u)\} .
$$


Using $\mathcal{C}$, take the functions $L$ and $l$ respectively in the primal function (2) to be

$$
L(t, x, y, z, h)= \begin{cases}\inf _{u \in \mathcal{C}} G(t, x, y, u), & \text { if } \mathcal{C} \neq \emptyset, \\ \infty, & \text { otherwise }\end{cases}
$$

and

$$
l(x)=g(x)
$$

With $L$ and $l$ so defined, the control problem (31) becomes a particular stochastic convex problem (3).

If $r=n$ and if $b$ and $\sigma$ are both affine functions of $(x, y, u)$, the corresponding $\mathcal{C}$ defined above contains a single element, determined by $n(1+m)$ linear equations, if it is not empty. Then, the expression for the corresponding $L$ simplifies. Moreover, under appropriate assumptions on the coefficients of these affine functions and on $G$ and $g$, including the convexity of $G$ and $g$, it can be checked that the corresponding problem (3) satisfies the required Assumptions I, II \& III. The following example demonstrates that this connection makes it possible to express an optimal control $\bar{u}$ of (31) in terms of solutions to the corresponding dual problem.

Example 4.1. For simplicity, we set $n=m=1$. Suppose that $\mathbb{U}=\mathbb{R}$; that $b(t, x, y, u)$ and $\sigma(t, x, y, u)$ in (30) are given by

$$
\left\{\begin{array}{l}
b(t, x, y, u)=a_{1}(t) x+b_{1}(t) y+c_{1}(t) u \\
\sigma(t, x, y, u)=a_{2}(t) x+b_{2}(t) y+c_{2}(t) u
\end{array}\right.
$$

where $a_{i}, b_{i}$ and $c_{i}$ are given $\mathbb{R}$-valued continuous functions on $[0, T]$ and $c_{1}(t)^{2}+c_{2}(t)^{2} \neq$ 0 , for all $t \in[0, T]$; and that

$$
G(t, x, y, u)=\frac{1}{2} c_{3}(t) u^{2} \quad \text { and } \quad g(x)=a_{3} x^{2},
$$

where $c_{3}:[0, T] \rightarrow \mathbb{R}_{+}$is continuous and $a_{3}>0$ is a constant. Then, Assumptions I, II \& III are satisfied and the corresponding stochastic convex primal problem (3) is

$$
\inf _{X \in \mathbb{X}}\left\{\mathrm{E}\left[\int_{0}^{T} \frac{1}{2} c_{3}(t) u^{2}(t) d t\right]+\mathrm{E}[g(X(T))]\right\},
$$

subject to

$$
\left\{\begin{array}{l}
\dot{X}(t)=a_{1}(t) X(t)+b_{1}(t) X_{\delta}(t)+c_{1}(t) u(t) \\
H_{X}(t)=a_{2}(t) X(t)+b_{2}(t) X_{\delta}(t)+c_{2}(t) u(t)
\end{array} \quad d \mathrm{P} \otimes d t\right. \text {-a.s. }
$$


where $X$ is identified with $\left(\dot{X}, H_{X}\right) \in \mathbb{X}$ via (1).

For $P$ identified with $\left(P_{T}, \dot{P}\right) \in \mathbb{P}$ via $(12)$, since $l(x)=g(x)$,

$$
l^{*}\left(-P_{T}\right)=\frac{P_{T}^{2}}{4 a_{3}} .
$$

Similarly, the expression (33) for $L$ gives that

$$
\begin{gathered}
L^{*}\left(t, \dot{P}(t)-\mathrm{E}\left[\dot{Q}(t+\delta) I_{[0, T-\delta]}(t) \mid \mathcal{F}(t)\right], \dot{Q}(t), P(t), H_{P}(t)\right) \\
=\sup _{(x, y) \in \mathbb{R}^{2}}\left\{x\left(\dot{P}(t)-\mathrm{E}\left[\dot{Q}(t+\delta) I_{[0, T-\delta]}(t) \mid \mathcal{F}(t)\right]\right)+y \dot{Q}(t)\right. \\
\left.\quad+\left(a_{1}(t) x+b_{1}(t) y\right) P(t)+\left(a_{2}(t) x+b_{2}(t) y\right) H_{P}(t)\right\} \\
+\sup _{u \in \mathbb{R}}\left\{u\left(c_{1}(t) P(t)+c_{2}(t) H_{P}(t)\right)-\frac{1}{2} c_{3}(t) u^{2}\right\}
\end{gathered}
$$

for $(P, \dot{Q}) \in \mathbb{P} \times \mathbb{L}_{\mathcal{F}}^{21}$, where $H_{P}$ is specified by $P$ via (13) and $P_{T}=-2 a_{3} X(T)$ by (28). To find an explicit expression for $L^{*}$ in (37), we take the derivatives, with respect to $x$ and $y$ respectively, of the function within the first bracket on the right-hand-side of (37). We obtain that the corresponding derivatives are zero if and only if

$$
\left\{\begin{array}{l}
\dot{P}(t)=\mathrm{E}\left[\dot{Q}(t+\delta) I_{[0, T-\delta]}(t) \mid \mathcal{F}(t)\right]-a_{1}(t) P(t)-a_{2}(t) H_{P}(t) \\
\dot{Q}(t)=-b_{1}(t) P(t)-b_{2}(t) H_{P}(t) .
\end{array}\right.
$$

Similarly, taking the derivative, with respect to $u$, of the function within the second bracket on the right-hand-side of (37), we see that the corresponding derivative is zero if and only if

$$
u=\frac{1}{c_{3}(t)}\left\{c_{1}(t) P(t)+c_{2}(t) H_{P}(t)\right\}
$$

This gives that

$$
\begin{aligned}
& L^{*}\left(t, \dot{P}(t)-\mathrm{E}\left[\dot{Q}(t+\delta) I_{[0, T-\delta]}(t) \mid \mathcal{F}(t)\right], \dot{Q}(t), P(t), H_{P}(t)\right) \\
= & \begin{cases}\frac{1}{2 c_{3}(t)}\left\{c_{1}(t) P(t)+c_{2}(t) H_{P}(t)\right\}^{2}, & \text { if (38) holds, } \\
\infty, & \text { otherwise. }\end{cases}
\end{aligned}
$$

Now, if $(u, X, P, \dot{Q})$ is such that $u$ satisfies $(39) ; X$ is identified with $\left(\dot{X}, H_{X}\right)$, where $\left(\dot{X}, H_{X}\right)$ is defined by $(36)$; and $P$ is identified with $\left(-2 a_{3} X(T), \dot{P}\right)$, where $(\dot{P}, \dot{Q})$ satisfies (38), then it can be verified that the two equalities in Theorem 3.2(iii) hold for such $(u, X, P, \dot{Q})$. Thus, by Theorem $3.2, u$ is an optimal control for the control problem corresponding to (35). 
Note that, if we replace $g$ in Example 4.1 by $g(x)=a_{3} x$, the above argument and derivation can be repeated except that $l^{*}\left(-P_{T}\right)$ becomes zero. Then, the modification to the result is that $P_{T}=-a_{3}$ rather than $-2 a_{3} X(T)$. Since $P_{T}$ becomes a constant, the corresponding $H_{P}$ is zero and $P$ is deterministic (see [4]). Thus, the corresponding optimal $u$ is also deterministic and given by $u=c_{1}(t) P(t) / c_{3}(t)$.

For more general $b, \sigma, G$ and $g$, to ensure that the set $\mathcal{C}$ is not empty and that the link of the stochastic control problem (31) to the stochastic convex problem (3) enables us to apply Theorem 3.2, we make the following assumptions.

Hypothesis I. The functions $b$ and $\sigma$ are continuous with respect to $(t, u) \in[0, T] \times \mathbb{U}$; and are Lipschitz continuous with respect to $(x, y) \in \mathbb{R}^{n} \times \mathbb{R}^{n}$ with the Lipschitz constant independent of $(t, u) \in[0, T] \times \mathbb{U}$. Moreover, there exists a constant $c_{1}>0$ such that for $f(t, x, y, u)=b(t, x, y, u)$ or $\sigma(t, x, y, u)$,

$$
|f(t, 0,0, u)| \leq c_{1}, \quad \forall(t, u) \in[0, T] \times \mathbb{U} .
$$

Hypothesis II. $g$ is a convex function of $x$. Moreover, there exist constants $c_{2} \in \mathbb{R}$ and $c_{3}>0$ such that

$$
\begin{cases}c_{2} \leqslant G(t, x, y, u) \leqslant c_{3}\left(1+|x|^{2}+|y|^{2}\right), & \forall(t, x, y, u) \in[0, T] \times \mathbb{R}^{n} \times \mathbb{R}^{n} \times \mathbb{U}, \\ c_{2} \leqslant g(x) \leqslant c_{3}\left(1+|x|^{2}\right), & \forall x \in \mathbb{R}^{n} .\end{cases}
$$

We now show that, under these two hypotheses, $L$ and $l$ defined by (33) and (34) satisfy Assumptions I, II \& III, except for the convexity requirement for $L$.

It is straightforward to verify that, under these hypotheses, $L$ and $l$ so defined are lower semi-continuous and are not identically $\infty$. Moreover, the argument for the Markovian control problems in [1, page 393] can be generalized to show that the conditions in (b) of Assumption I for $L$ and $l$ are satisfied. Thus, except for the required convexity of $L$, all conditions in Assumption I are satisfied by $L$ and $l$. We now show, in the following proposition, that the remaining two assumptions are also satisfied.

Proposition 4.1. Under Hypotheses I \& II, the functions $L$ and $l$ defined respectively by (33) and (34) satisfy Assumptions II \& III.

Proof. By Hypothesis II, $G$ and $g$ are bounded below, which implies that $L$ and $l$ are bounded below. Hence, $L$ and $l$ satisfy Assumption II. 
On the other hand, Hypothesis I implies that, for any given $\hat{u} \in \mathcal{U}$, there exists a unique $\mathcal{F}(t)$-adapted solution $\hat{X}$ to (30) such that $\hat{X}, \hat{X}_{\delta} \in \mathbb{L}_{\mathcal{F}}^{22}$ (see [4, Theorem 2.2]). Hence,

$$
\hat{\mathcal{C}}=\mathcal{C}\left(t, \hat{X}(t), \hat{X}_{\delta}(t), \dot{\hat{X}}(t), H_{\hat{X}}(t)\right) \neq \emptyset
$$

where

$$
\left\{\begin{array}{l}
\dot{\hat{X}}(t)=b\left(t, \hat{X}(t), \hat{X}_{\delta}(t), \hat{u}(t)\right) \\
H_{\hat{X}}(t)=\sigma\left(t, \hat{X}(t), \hat{X}_{\delta}(t), \hat{u}(t)\right)
\end{array} \quad d \mathrm{P} \otimes d t\right. \text {-a.s. }
$$

In particular, by the Cauchy-Schwarz Inequality, (40) and the fact that $\hat{X}, \hat{X}_{\delta} \in \mathbb{L}_{\mathcal{F}}^{22}$ together imply that $\dot{\hat{X}} \in \mathbb{L}_{\mathcal{F}}^{21}$ and $H_{\hat{X}} \in \mathbb{L}_{\mathcal{F}}^{22}$.

Since $\hat{\mathcal{C}}$ is not empty and since Hypothesis II holds, we have

$$
\begin{aligned}
& L\left(t, \hat{X}(t), \hat{X}_{\delta}(t), \dot{\hat{X}}(t), H_{\hat{X}}(t)\right) \\
= & \inf _{u \in \hat{\mathcal{C}}} G\left(t, \hat{X}(t), \hat{X}_{\delta}(t), u\right) \leqslant c_{3}\left(1+|\hat{X}(t)|^{2}+\left|\hat{X}_{\delta}(t)\right|^{2}\right), \quad d \mathrm{P} \otimes d t \text {-a.s. }
\end{aligned}
$$

and

$$
l(\hat{X}(T))=g(\hat{X}(T)) \leqslant c_{3}\left(1+|\hat{X}(T)|^{2}\right), \quad d \mathrm{P} \text {-a.s. }
$$

Thus, taking $\tau_{2}$ and $\theta_{2}$ in Assumption III to be $c_{3}\left(1+|\hat{X}(t)|^{2}+\left|\hat{X}_{\delta}(t)\right|^{2}\right)$ and $c_{3}(1+$ $\left.|\hat{X}(T)|^{2}\right)$ respectively, we see that $\tau_{2}$ and $\theta_{2}$ satisfy the required conditions, so that $L$ and $l$ satisfy Assumption III.

Turning to the convexity of $L$, which is not guaranteed by Hypothesis I \& II, but is required for Assumption I, the following proposition gives a sufficient condition for it to hold.

Proposition 4.2. Let $\mathcal{H}:[0, T] \times \mathbb{R}^{n} \times \mathbb{R}^{n} \times \mathbb{U} \times \mathbb{R}^{n} \times \mathbb{R}^{n \times m} \rightarrow \mathbb{R}$ be defined by

$$
\mathcal{H}(t, x, y, u, p, h)=\langle b(t, x, y, u), p\rangle+\langle\sigma(t, x, y, u), h\rangle-G(t, x, y, u) .
$$

If $\mathcal{H}$ is concave with respect to $(x, y, u)$, then $L$ defined by (33) is a convex function with respect to $(x, y, z, h)$.

Proof. Let

$$
\tilde{L}(t, x, y, z, h)=\inf _{u \in \mathbb{U}}\left\{\sup _{\left(p, h_{p}\right) \in \mathbb{R}^{n} \times \mathbb{R}^{n \times m}}\left\{\left\langle(z, h),\left(p, h_{p}\right)\right\rangle-\mathcal{H}\left(t, x, y, u, p, h_{p}\right)\right\}\right\} .
$$


Then, the expression (41) for $\mathcal{H}$ gives

$$
\begin{aligned}
\tilde{L}(t, x, y, z, h) & =\inf _{u \in \mathbb{U}}\{G(t, x, y, u) \\
& \left.+\sup _{\left(p, h_{p}\right) \in \mathbb{R}^{n} \times \mathbb{R}^{n \times m}}\left\{\left\langle(z-b(t, x, y, u), h-\sigma(t, x, y, u)),\left(p, h_{p}\right)\right\rangle\right\}\right\} .
\end{aligned}
$$

For $\mathcal{C}=\mathcal{C}(t, x, y, z, h)$ as defined in $(32)$, if $\mathcal{C}=\emptyset$, then $(z-b(t, x, y, u), h-\sigma(t, x, y, u)) \neq$ $(0,0)$ and so the supremum in (43) is $\infty$, which implies that $\tilde{L}=\infty$. Otherwise, $\tilde{L}(t, x, y, z, h)=\inf _{u \in \mathcal{C}} G(t, x, y, u)$. Hence, $\tilde{L}=L$, where $L$ is defined by (33).

Since $\mathcal{H}$ is linear in $\left(p, h_{p}\right),\left\langle(z, h),\left(p, h_{p}\right)\right\rangle-\mathcal{H}\left(t, x, y, u, p, h_{p}\right)$ is convex in $\left(u, p, h_{p}\right)$ by the assumption. Then, the order of the supremum and the infimum on the righthand-side of (42) can be exchanged (see [17, Corollary 37.2.2]) so that

$$
L(t, x, y, z, h)=\sup _{\left(p, h_{p}\right) \in \mathbb{R}^{n} \times \mathbb{R}^{n \times m}}\left\{\left\langle(z, h),\left(p, h_{p}\right)\right\rangle-\hat{\mathcal{H}}\left(t, x, y, p, h_{p}\right)\right\},
$$

where $\hat{\mathcal{H}}\left(t, x, y, p, h_{p}\right)=\sup _{u \in \mathbb{U}} \mathcal{H}\left(t, x, y, u, p, h_{p}\right)$. Since $\mathbb{U}$ is a convex set, it is easy to check that $\hat{\mathcal{H}}$ is concave in $(x, y)$ and convex in $\left(p, h_{p}\right)$. Therefore, (44) implies that $L$ is convex in $(x, y, z, h)$ as required.

To end this subsection, we use an example to demonstrate that there are indeed stochastic control problems where at least one of $b$ and $\sigma$ is not an affine function of $(x, y, u)$, but which can be reformulated as stochastic convex problems studied in the previous sections.

Example 4.2. We assume that $n=m=r=1$. Suppose that $\mathbb{U}=(0,2 \pi]$; that

$$
b(t, x, y, u)=\sin (x+y+u), \quad \sigma(t, x, y, u)=y
$$

and that $G(t, x, y, u)=|x+\sin (x+y+u)|$ and $g(x)=x^{2}$. The functions so chosen satisfy Hypotheses I and II. Moreover,

$$
\mathcal{C}(t, x, y, z, h)=\{u \in(0,2 \pi] \mid z=\sin (x+y+u) \text { and } h=y\}
$$

and $\mathcal{C}(t, x, y, z, h) \neq \emptyset$ if and only if $|z| \leqslant 1$. This gives that

$$
L(t, x, y, z, h)= \begin{cases}|x+z|, & \text { if }|z| \leqslant 1 \text { and } h=y \\ \infty, & \text { otherwise. }\end{cases}
$$


Clearly, $L$ is a convex function of $(x, y, z, h)$. Hence, by Proposition 4.1, as well as the discussion prior to it, the stochastic control problem associated with $b, \sigma, G$ and $g$ defined here is transformed into a stochastic convex problem of the type studied in the previous sections.

\subsection{Stochastic maximum principles}

We now use Theorem 3.2, in particular conditions (27) and (28), to derive the sufficient conditions for optimality, as well as the expressions for the Hamiltonian and associated adjoint equation, for the problem (31).

For the control problem (31), define the processes $\left(P, H_{P}\right) \in \mathbb{L}_{\mathcal{F}}^{2 \infty} \times \mathbb{L}_{\mathcal{F}}^{22}$ by the following anticipated BSDE

$$
\left\{\begin{aligned}
d P(t)=- & \left\{\frac{\partial \mathcal{H}}{\partial x}(t)+\mathrm{E}\left[\frac{\partial \mathcal{H}}{\partial y}(t+\delta) I_{[0, T-\delta]}(t) \mid \mathcal{F}(t)\right]\right\} d t \\
& +H_{P}(t) d B(t) \quad t \in[0, T), \\
P(T)=- & \frac{\partial g}{\partial x}(X(T))
\end{aligned}\right.
$$

where $\mathcal{H}$ is defined by (41), where we have used the shorthand notation

$$
\frac{\partial \mathcal{H}}{\partial x}(t)=\frac{\partial \mathcal{H}}{\partial x}\left(t, X(t), X_{\delta}(t), u(t), P(t), H_{P}(t)\right)
$$

and similarly for the partial derivative $\frac{\partial \mathcal{H}}{\partial y}(t+\delta)$, and where we assume the necessary differentiability of $\mathcal{H}$.

Note that, if $\delta=0$ so that there is no delay in the model, $\mathcal{H}$ defined by (41) is independent of $y$, corresponding to $X_{\delta}$. Then, the corresponding $\mathcal{H}$ and equation (45) are termed as the (stochastic) Hamiltonian (function) and the adjoint equation due to their link with the deterministic cases (see [21, Chapter 3]). We adopt them for our model and the following result justifies this usage.

Theorem 4.1. Assume that Hypotheses I \& II hold and that L defined by (33) is convex with respect to $(x, y, z, h)$. In addition, assume that $\mathbb{U}$ is compact and that the functions $b, \sigma$ and $G$ are continuously differentiable with respect to $(x, y)$ and that $g$ is continuously differentiable with respect to $x$. Suppose that $\bar{X} \in \mathbb{X}$ and $(\bar{P}, \dot{\bar{Q}}) \in \mathbb{P} \times \mathbb{L}_{\mathcal{F}}^{21}$ together satisfy (27) and (28) with $L$ and $l$ being defined by (33) and (34) respectively. Then, it is necessary that there exists a $\bar{u} \in \mathcal{U}$ realizing (31). Moreover, 
(i) $\bar{X}$ is the unique strong solution of the controlled SDDE (30) with $u$ in the functions $b$ and $\sigma$ replaced by $\bar{u}$;

(ii) $\left(\bar{P}, H_{\bar{P}}\right)$ is a solution of the adjoint equation (45) with $\left(X, X_{\delta}, u\right)$ replaced by $\left(\bar{X}, \bar{X}_{\delta}, \bar{u}\right)$, where $H_{\bar{P}}$ is specified by $\bar{P}$ via (13);

(iii) $d \mathrm{P} \otimes d t$-a.s.,

$$
\begin{aligned}
& \mathcal{H}\left(t, \bar{X}(t), \bar{X}_{\delta}(t), \bar{u}(t), \bar{P}(t), H_{\bar{P}}(t)\right) \\
= & \max _{u \in \mathbb{U}} \mathcal{H}\left(t, \bar{X}(t), \bar{X}_{\delta}(t), u, \bar{P}(t), H_{\bar{P}}(t)\right),
\end{aligned}
$$

where $\mathcal{H}$ is defined by (41).

Proof. Given that the control problem (31) has been reformulated as the corresponding primal problem (3), with $L$ defined by (33) being convex, Assumptions I, II \& III are satisfied by the reformulated problem (3). Moreover, under the given conditions, it follows from Theorem 3.2(ii) that $\bar{X}$ is a solution of the corresponding primal problem (3).

By (27),

$$
\begin{aligned}
& L^{*}\left(t, \dot{\bar{P}}(t)-\mathrm{E}\left[\dot{\bar{Q}}(t+\delta) I_{[0, T-\delta]}(t) \mid \mathcal{F}(t)\right], \dot{\bar{Q}}(t), \bar{P}(t), H_{\bar{P}}(t)\right) \\
= & \left\langle\bar{X}(t), \dot{\bar{P}}(t)-\mathrm{E}\left[\dot{\bar{Q}}(t+\delta) I_{[0, T-\delta]}(t) \mid \mathcal{F}(t)\right]\right\rangle \\
& +\left\langle\bar{X}_{\delta}(t), \dot{\bar{Q}}(t)\right\rangle+\left\langle\left(\dot{\bar{X}}(t), H_{\bar{X}}(t)\right),\left(\bar{P}(t), H_{\bar{P}}(t)\right)\right\rangle \\
& -L\left(t, \bar{X}(t), \bar{X}_{\delta}(t), \dot{\bar{X}}(t), H_{\bar{X}}(t)\right), \quad d \mathrm{P} \otimes d t \text {-a.s. },
\end{aligned}
$$

where $\left(\dot{\bar{X}}, H_{\bar{X}}\right)$ is defined by (1) with $X$ replaced by $\bar{X}$ and where $H_{\bar{P}}$ is specified by $\bar{P}$ via (13). On the other hand, using the expression (33) for $L$ and using the definition of conjugation functions, $L^{*}$ in (47) can also be expressed, in terms of $b, \sigma$ and $G$, as

$$
\begin{aligned}
& L^{*}\left(t, \dot{\bar{P}}(t)-\mathrm{E}\left[\dot{\bar{Q}}(t+\delta) I_{[0, T-\delta]}(t) \mid \mathcal{F}(t)\right], \dot{\bar{Q}}(t), \bar{P}(t), H_{\bar{P}}(t)\right) \\
&=\sup _{(x, y) \in \mathbb{R}^{n} \times \mathbb{R}^{n}} \max _{u \in \mathbb{U}}\{\left\langle\dot{\bar{P}}(t)-\mathrm{E}\left[\dot{\bar{Q}}(t+\delta) I_{[0, T-\delta]}(t) \mid \mathcal{F}(t)\right], x\right\rangle \\
&+\langle\dot{\bar{Q}}(t), y\rangle+\langle\bar{P}(t), b(t, x, y, u)\rangle \\
&\left.+\left\langle H_{\bar{P}}(t), \sigma(t, x, y, u)\right\rangle-G(t, x, y, u)\right\} .
\end{aligned}
$$

Since $\mathbb{U}$ is compact, (47) and (48) together imply that, for the given $\bar{X}$ and $(\bar{P}, \dot{\bar{Q}})$, it 
is necessary that there is a $\bar{u} \in \mathcal{U}$ such that $\left(\dot{\bar{X}}, H_{\bar{X}}\right)$ has the expression

$$
\left\{\begin{array}{l}
\dot{\bar{X}}(t)=b\left(t, \bar{X}(t), \bar{X}_{\delta}(t), \bar{u}(t)\right) \\
H_{\bar{X}}(t)=\sigma\left(t, \bar{X}(t), \bar{X}_{\delta}(t), \bar{u}(t)\right)
\end{array} \quad d \mathrm{P} \otimes d t\right. \text {-a.s. }
$$

and that the 'sup max' in (48) is attained at $\left(\bar{X}(t), \bar{X}_{\delta}(t), \bar{u}(t)\right), d \mathrm{P} \otimes d t$-a.s. Noting the fact that $\bar{X}$ is a solution of the corresponding primal problem (3), (49) implies that $\bar{u}$ is an optimal control for the control problem (31) and that $\bar{X}$ is the unique solution to (30) with $u$ replaced by $\bar{u}$, i.e. $(i)$ holds.

Using the expression (41) for $\mathcal{H}$, it also follows from (47) and (48) that

$$
\mathcal{H}\left(t, \bar{X}(t), \bar{X}_{\delta}(t), \bar{u}(t), \bar{P}(t), H_{\bar{P}}(t)\right)=\max _{u \in \mathbb{U}} \mathcal{H}\left(t, \bar{X}(t), \bar{X}_{\delta}(t), u, \bar{P}(t), H_{\bar{P}}(t)\right)
$$

i.e. (iii) holds.

To show (ii), we note first that, using the expression (41) for $\mathcal{H}$ again, (47) and (48) together imply further that, $d \mathrm{P} \otimes d t$-a.s.

$$
\begin{aligned}
&\left\langle\bar{X}(t), \dot{\bar{P}}(t)-\mathrm{E}\left[\dot{\bar{Q}}(t+\delta) I_{[0, T-\delta]}(t) \mid \mathcal{F}(t)\right]\right\rangle \\
&+\left\langle\bar{X}_{\delta}(t), \dot{\bar{Q}}(t)\right\rangle+\mathcal{H}\left(t, \bar{X}(t), \bar{X}_{\delta}(t), \bar{u}(t), \bar{P}(t), H_{\bar{P}}(t)\right) \\
&=\max _{(x, y) \in \mathbb{R}^{n} \times \mathbb{R}^{n}}\left\{\left\langle x, \dot{\bar{P}}(t)-\mathrm{E}\left[\dot{\bar{Q}}(t+\delta) I_{[0, T-\delta]}(t) \mid \mathcal{F}(t)\right]\right\rangle\right. \\
&\left.+\langle y, \dot{\bar{Q}}(t)\rangle+\mathcal{H}\left(t, x, y, \bar{u}(t), \bar{P}(t), H_{\bar{P}}(t)\right)\right\} .
\end{aligned}
$$

Since $b, \sigma$ and $G$ are differentiable with respect to $(x, y)$, by taking the derivatives with respect to $x$ and $y$ of the function within the bracket on the right-hand-side of the above equation, the fact that the maximum in the above equation is attained at $\left(\bar{X}(t), \bar{X}_{\delta}(t)\right), d \mathrm{P} \otimes d t$-a.s., implies that

$$
\dot{\bar{P}}(t)=-\frac{\partial \overline{\mathcal{H}}}{\partial x}(t)+\mathrm{E}\left[\dot{\bar{Q}}(t+\delta) I_{[0, T-\delta]}(t) \mid \mathcal{F}(t)\right], \quad d \mathrm{P} \otimes d t \text {-a.s. }
$$

and

$$
\dot{\bar{Q}}(t)=-\frac{\partial \overline{\mathcal{H}}}{\partial y}(t), \quad d \mathrm{P} \otimes d t \text {-a.s. }
$$

where $\overline{\mathcal{H}}(t)=\mathcal{H}\left(t, \bar{X}(t), \bar{X}_{\delta}(t), \bar{u}(t), \bar{P}(t), H_{\bar{P}}(t)\right)$. Replacing $\dot{\bar{Q}}$ in (51) using (52) gives

$$
\dot{\bar{P}}(t)=-\frac{\partial \overline{\mathcal{H}}}{\partial x}(t)-\mathrm{E}\left[\frac{\partial \overline{\mathcal{H}}}{\partial y}(t+\delta) I_{[0, T-\delta]}(t) \mid \mathcal{F}(t)\right], \quad d \mathrm{P} \otimes d t \text {-a.s. }
$$


Similarly, by (28), we have

$$
l^{*}\left(-\bar{P}_{T}\right)=\left\langle-\bar{P}_{T}, \bar{X}(T)\right\rangle-l(\bar{X}(T)), \quad d \mathrm{P} \text {-a.s. }
$$

Since $l^{*}$ is the conjugate convex function of $l$ and since $l=g$, the above, together with the definitions of conjugate functions, implies that

$$
\left\langle-\bar{P}_{T}, \bar{X}(T)\right\rangle-g(\bar{X}(T))=\sup _{x \in \mathbb{R}^{n}}\left\{\left\langle x,-\bar{P}_{T}\right\rangle-g(x)\right\}, \quad d \mathrm{P} \text {-a.s. }
$$

Taking the derivative, with respect to $x$, of the function within the bracket on the right-hand-side of the above equation, we see that $\bar{P}_{T}$ must satisfy the condition that

$$
\bar{P}_{T}=-\frac{\partial g}{\partial x}(\bar{X}(T)), \quad d \mathrm{P} \text {-a.s. }
$$

Now, since $\bar{P}=\left(\bar{P}_{T}, \dot{\bar{P}}\right) \in \mathbb{P}$, using (13), (53) and (54) gives that

$$
\begin{aligned}
& \bar{P}(t) \\
= & -\frac{\partial g}{\partial x}(\bar{X}(T))+\int_{t}^{T}\left\{\mathrm{E}\left[\frac{\partial \overline{\mathcal{H}}}{\partial y}(s+\delta) I_{[0, T-\delta]}(s) \mid \mathcal{F}(s)\right]+\frac{\partial \overline{\mathcal{H}}}{\partial x}(s)\right\} d s \\
& -\int_{t}^{T} H_{\bar{P}}(s) d B(s), \quad d \mathrm{P} \text {-a.s. }
\end{aligned}
$$

i.e. (ii) holds.

Note that, rather than defining them, the proof of the above theorem uses the techniques of conjugate duality to derive the Hamiltonian $\mathcal{H}$ and the associated adjoint equation for the problem (31). If $\delta=0$, the Hamiltonian $\mathcal{H}$ is independent of $y$, which corresponds to the delayed variable, and then the adjoint equation (45) reduces to a classic BSDE studied in [21, Chapter 3].

Recall that, by Proposition 4.2, the concavity condition on the Hamiltonian $\mathcal{H}$ implies the required convexity of $L$. Under such a concavity condition on $\mathcal{H}$, the proof of Theorem 4.1 can be modified to give the following sufficient maximum principle.

Theorem 4.2. In addition to Hypotheses I \& II, we assume further that the functions $b, \sigma$ and $G$ are continuously differentiable with respect to $(x, y)$, that $g$ is continuously differentiable with respect to $x$ and that $\mathcal{H}(t, x, y, u, p, h)$ is concave with respect to $(x, y, u)$. Let $\bar{u} \in \mathcal{U}, \bar{X}$ be the solution to the controlled SDDE (30) associated with $\bar{u}$, and $\left(\bar{P}, H_{\bar{P}}\right)$ be the solution to the adjoint equation (45) associated with $(\bar{u}, \bar{X})$. If $(\bar{u}, \bar{X}, \bar{P})$ satisfies (46), then $\bar{u}$ is an optimal solution for the control problem (31). 
Proof. For the given $\bar{u}, \bar{X}$ and $\left(\bar{P}, H_{\bar{P}}\right)$, we have $\bar{P}_{T}, \dot{\bar{P}}$ and $\dot{\bar{Q}}$ respectively defined by (54), (51) and (52). Under the given conditions, $\bar{P}_{T} \in \mathbb{L}^{2}, \dot{\bar{P}} \in \mathbb{L}_{\mathcal{F}}^{21}$ and $\dot{\bar{Q}} \in \mathbb{L}_{\mathcal{F}}^{21}$. It follows from (13) and from the uniqueness of the martingale representation that $\bar{P}$ is identified with $\left(\bar{P}_{T}, \dot{\bar{P}}\right) \in \mathbb{P}$ via (12). Furthermore, given that $(\bar{u}, \bar{X}, \bar{P})$ satisfies (46), the argument in the proof of Theorem 4.1, together with the given concavity of $\mathcal{H}$, shows that, for such $(\bar{P}, \dot{\bar{Q}})$, the 'sup max' in $(48)$ is attained at $\left(\bar{X}, \bar{X}_{\delta}, \bar{u}\right)$, i.e. (27) holds. Similarly, the proof of Theorem 4.1 also shows that (28) holds. Thus, the required result follows from Theorem 3.2.

Comparing with [4], [12] and [14], the above sufficient stochastic maximum principle is proved using the method of conjugate duality, for which we require Hypotheses I \& II. Otherwise, the other conditions set in the theorem are similar to those required in [4, Theorem 3.2] and the result is similar to those in [4], [12] and [14] when their models are restricted to ours.

\section{The inclusion of exponential moving average delay}

The methods and results obtained in the preceding sections can be extended to include an exponential moving average delay, in addition to the discrete delay $X_{\delta}$, in the model. That is, the continuous $\mathcal{F}(t)$-adapted state process $X$ is described by the controlled SDDE

$$
\left\{\begin{aligned}
d X(t)= & b\left(t, X(t), X_{a}(t), X_{\delta}(t), u(t)\right) d t & & \\
& +\sigma\left(t, X(t), X_{a}(t), X_{\delta}(t), u(t)\right) d B(t), & & t \in(0, T], \\
X(t)= & x_{0}(t), & & t \in[-\delta, 0],
\end{aligned}\right.
$$

where $x_{0}, X_{\delta}, \delta$ and $u$ are as defined before and $X_{a}$ denotes the exponential moving average delay of $X$ given by

$$
X_{a}(t)=\int_{-\delta}^{0} e^{\lambda s} X(t+s) d s, \quad t \in[0, T] .
$$

The functions $G$ and $g$ may also depend respectively on $X_{a}$ and $X_{a}(T)$, and the associated optimal control problem is to find $\bar{u} \in \mathcal{U}$ realizing

$$
\inf _{u \in \mathcal{U}} J_{a}(u)
$$


where

$$
J_{a}(u)=\mathbb{E}\left[\int_{0}^{T} G\left(t, X(t), X_{a}(t), X_{\delta}(t), u(t)\right) d t+g\left(X(T), X_{a}(T)\right)\right] .
$$

Note that this type of stochastic control problem with delay was studied in [13], where the authors obtain a sufficient condition for the maximum principle using methods of stochastic calculus.

As in [7], we introduce the state process $V: \Omega \times[0, T] \rightarrow \mathbb{R}^{n}$ defined by

$$
\left\{\begin{array}{l}
d V(t)=\left\{X(t)-\lambda V(t)-e^{-\lambda \delta} X_{\delta}(t)\right\} d t, \quad t \in[0, T], \\
V(0)=v_{0}=\int_{-\delta}^{0} e^{\lambda s} x_{0}(s) d s
\end{array}\right.
$$

Then, $V(t)=X_{a}(t)$ and so the combined SDDE for $W=(X, V)$, given by (55) with $X_{a}$ replaced by $V$ and (57), is equivalent to the original controlled SDDE (55) for $X$. In terms of this new combined SDDE, the stochastic optimal control problem associated with (55) becomes a stochastic optimal control problem with discrete delay, where its drift and diffusion coefficients are independent of $V_{\delta}$.

To derive the adjoint equations and the stochastic maximum principle for the stochastic optimal control problem associated with (55), and to improve the results in $[13,14]$, we modify our previous conjugate duality approach to extend it to $W=(X, V)$. For this, in addition to $X \in \mathbb{X}$, we identify $\left(\dot{V}, H_{V}\right) \in \mathbb{X}$ with the continuous $\mathcal{F}(t)$ adapted stochastic process $V: \Omega \times[0, T] \rightarrow \mathbb{R}^{n}$ defined by

$$
V(t)=v_{0}+\int_{0}^{t} \dot{V}(s) d s+\int_{0}^{t} H_{V}(s) d B(s)
$$

in a similar fashion to the identification of $X$ with $\left(\dot{X}, H_{X}\right) \in \mathbb{X}$. At the same time, take $L_{a}$ and $l_{a}$ to be modifications of $L$ and $l$ in Section 2, so that they depend also on $\left(V, \dot{V}, H_{V}\right)$ and on $V(T)$ respectively. Then, the corresponding stochastic convex problem with discrete delay is to find $(\bar{X}, \bar{V}) \in \mathbb{X} \times \mathbb{X}$ realizing

$$
\inf _{(X, V) \in \mathbb{X} \times \mathbb{X}} \Phi_{a}(X, V)
$$

where

$$
\Phi_{a}(X, V)=I_{L_{a}}\left(X, V, X_{\delta}, \dot{X}, \dot{V}, H_{X}, H_{V}\right)+J_{l_{a}}(X(T), V(T)) .
$$

Adapting the arguments in Section 3 , in addition to $P=\left(P_{T}, \dot{P}\right) \in \mathbb{P}$, we require another continuous $\mathcal{F}(t)$-adapted stochastic process $P^{a}$ to pair with $V \in \mathbb{X}$, where 
$P^{a}: \Omega \times[0, T] \rightarrow \mathbb{R}^{n}$ is identified with $\left(P_{T}^{a}, \dot{P}^{a}\right) \in \mathbb{P}$ in the same sense that $P$ is identified with $\left(P_{T}, \dot{P}\right)$ using (12). Assuming that $L_{a}$ and $l_{a}$ satisfy the appropriately modified Assumptions I, II \& III of Section 2, the argument for the proof of Theorem 3.1 can be used to obtain the dual problem to (58) to be realising

$$
\inf _{\left(P, P^{a}, \dot{Q}\right) \in \mathbb{P} \times \mathbb{P} \times \mathbb{L}_{\mathcal{F}}^{21}} \Psi_{a}\left(P, P^{a}, \dot{Q}\right),
$$

where

$$
\begin{aligned}
& \Psi_{a}\left(P, P^{a}, \dot{Q}\right) \\
= & I_{L_{a}^{*}}\left(\dot{P}-\mathrm{E}\left[\dot{Q}(\cdot+\delta) I_{[0, T-\delta]}(\cdot) \mid \mathcal{F}(\cdot)\right], \dot{P}^{a}, \dot{Q}, P, P^{a}, H_{P}, H_{P^{a}}\right) \\
& +J_{l_{a}^{*}}\left(-P_{T},-P_{T}^{a}\right)-\mathrm{E}\left[\int_{0}^{T}\left\langle\dot{Q}(t), x_{0}(t-\delta) I_{[0, \delta]}(t)\right\rangle d t\right] \\
& -\mathrm{E}\left[\int_{0}^{T}\left(\left\langle\dot{P}(t), x_{0}(0)\right\rangle+\left\langle\dot{P}^{a}(t), v_{0}\right\rangle\right) d t\right]+\mathrm{E}\left[\left\langle\left(P_{T}, P_{T}^{a}\right),\left(x_{0}(0), v_{0}\right)\right\rangle\right]
\end{aligned}
$$

and where $H_{P^{a}} \in \mathbb{L}_{\mathcal{F}}^{22}$ is obtained by applying the martingale representation theorem to $P^{a} \in \mathbb{P}$ as for $H_{P}$ obtained from $P$ via (13). Since the combined SDDE is independent of $V_{\delta}$, the inclusion of $P^{a}$ in $\Psi_{a}$ does not result in the dependence of $\Psi_{a}$ on an additional $Q^{a}$ as was the case for the inclusion of $Q$ in $\Psi$. The expression for $\Psi_{a}$ then enables us to modify the proof of Theorem 3.2 to obtain the following equivalent conditions for optimality of this new stochastic convex problem.

Theorem 5.1. For any given $(\bar{X}, \bar{V}) \in \mathbb{X} \times \mathbb{X}$ and $\left(\bar{P}, \bar{P}^{a}, \dot{\bar{Q}}\right) \in \mathbb{P} \times \mathbb{P} \times \mathbb{L}_{\mathcal{F}}^{21}$, the following three statements are equivalent:

(i)

$$
\Phi_{a}(\bar{X}, \bar{V})+\Psi_{a}\left(\bar{P}, \bar{P}^{a}, \dot{\bar{Q}}\right)=0 .
$$

(ii) $(\bar{X}, \bar{V})$ and $\left(\bar{P}, \bar{P}^{a}, \dot{\bar{Q}}\right)$ are respectively optimal solutions to the primal problem (58) and its dual problem (59), and

$$
\inf _{(X, V) \in \mathbb{X} \times \mathbb{X}} \Phi_{a}(X, V)=-\inf _{\left(P, P^{a}, \dot{Q}\right) \in \mathbb{P} \times \mathbb{P} \times \mathbb{L}_{\mathcal{F}}^{21}} \Psi_{a}\left(P, P^{a}, \dot{Q}\right) .
$$


(iii)

$$
\begin{aligned}
& L_{a}^{*}\left(t, \dot{\bar{P}}(t)-\mathrm{E}\left[\dot{\bar{Q}}(t+\delta) I_{[0, T-\delta]}(t) \mid \mathcal{F}(t)\right], \dot{\bar{P}}^{a}(t), \dot{\bar{Q}}(t), \bar{P}(t), \bar{P}^{a}(t), H_{\bar{P}}(t), H_{\bar{P}^{a}}(t)\right) \\
& +L_{a}\left(t, \bar{X}(t), \bar{V}(t), \bar{X}_{\delta}(t), \dot{\bar{X}}(t), \dot{\bar{V}}(t), H_{\bar{X}}(t), H_{\bar{V}}(t)\right)-\left\langle\dot{\bar{Q}}(t), \bar{X}_{\delta}(t)\right\rangle \\
& -\left\langle\dot{\bar{P}}(t)-\mathrm{E}\left[\dot{\bar{Q}}(t+\delta) I_{[0, T-\delta]}(t) \mid \mathcal{F}(t)\right], \bar{X}(t)\right\rangle-\left\langle\left(\bar{P}(t), H_{\bar{P}}(t)\right),\left(\dot{\bar{X}}(t), H_{\bar{X}}(t)\right)\right\rangle \\
& -\left\langle\dot{\bar{P}}^{a}(t), \bar{V}(t)\right\rangle-\left\langle\left(\bar{P}^{a}(t), H_{\bar{P}^{a}}(t)\right),\left(\dot{\bar{V}}(t), H_{\bar{V}}(t)\right)\right\rangle=0, \quad d \mathrm{P} \otimes d t-\text { a.s. }
\end{aligned}
$$

and

$$
l_{a}(\bar{X}(T), \bar{V}(T))+l_{a}^{*}\left(-\bar{P}_{T},-\bar{P}_{T}^{a}\right)+\left\langle\left(\bar{P}_{T}, \bar{P}_{T}^{a}\right),(\bar{X}(T), \bar{V}(T))\right\rangle=0, d \mathrm{P}-\text { a.s. }
$$

Returning to the optimal control problem (56), by adapting the technique for the proof of Theorem 4.1, we see similarly that Theorem 5.1 implies the following extension of Theorem 4.1 to have a sufficient condition for optimality of (56), involving the Hamiltonian $\mathcal{H}_{a}$ of the problem (56) defined by

$$
\begin{aligned}
\mathcal{H}_{a}\left(t, x, y, z, u, p, r, h_{p}, h_{r}\right)= & \langle b(t, x, y, z, u), p\rangle+\left\langle x-\lambda y-e^{-\lambda \delta} z, r\right\rangle \\
& +\left\langle\sigma(t, x, y, z, u), h_{p}\right\rangle-G(t, x, y, z, u),
\end{aligned}
$$

and associated adjoint equations.

Theorem 5.2. Under the modified conditions to those in Theorem 4.1, suppose that $(\bar{X}, \bar{V}) \in \mathbb{X} \times \mathbb{X}$ and $\left(\bar{P}, \bar{P}^{a}, \dot{\bar{Q}}\right) \in \mathbb{P} \times \mathbb{P} \times \mathbb{L}_{\mathcal{F}}^{21}$ together satisfy the two equalities given in Theorem 5.1(iii) with $L_{a}$ and $l_{a}$ being defined using $G$ and $g$ in a similar manner to that specified in Section 4. Then, it is necessary that there is a $\bar{u} \in \mathcal{U}$ realising (56). Moreover,

(i) $\bar{X}$ is the unique strong solution of the controlled SDDE (55) with $u$ in the functions $b$ and $\sigma$ replaced by $\bar{u}$;

(ii) $\left(\bar{P}, H_{\bar{P}}\right)$ and $\left(\bar{P}^{a}, H_{\bar{P}^{a}}\right)$ are solutions of the following adjoint equations with $\left(X, X_{a}, X_{\delta}, u\right)$ replaced by $\left(\bar{X}, \bar{X}_{a}, \bar{X}_{\delta}, \bar{u}\right)$ :

$$
\left\{\begin{aligned}
d P(t)=- & \left\{\frac{\partial \mathcal{H}_{a}}{\partial x}(t)+\mathrm{E}\left[\frac{\partial \mathcal{H}_{a}}{\partial z}(t+\delta) I_{[0, T-\delta]}(t) \mid \mathcal{F}(t)\right]\right\} d t \\
& +H_{P}(t) d B(t), \quad t \in[0, T), \\
P(T)=-\frac{\partial g}{\partial x}\left(X(T), X_{a}(T)\right) &
\end{aligned}\right.
$$


and

$$
\left\{\begin{array}{l}
d P^{a}(t)=-\frac{\partial \mathcal{H}_{a}}{\partial y}(t) d t+H_{P^{a}}(t) d B(t), \quad t \in[0, T], \\
P^{a}(T)=-\frac{\partial g}{\partial y}\left(X(T), X_{a}(T)\right)
\end{array}\right.
$$

where $H_{\bar{P}}$ and $H_{\bar{P}^{a}}$ are respectively specified by $\bar{P}$ and $\bar{P}^{a}$ via (13).

(iii) $d \mathrm{P} \otimes d t$-a.s.,

$$
\begin{aligned}
& \mathcal{H}_{a}\left(t, \bar{X}(t), \bar{X}_{a}(t), \bar{X}_{\delta}(t), \bar{u}(t), \bar{P}(t), \bar{P}^{a}(t), H_{\bar{P}}(t), H_{\bar{P}^{a}}(t)\right) \\
= & \max _{u \in \mathbb{U}} \mathcal{H}_{a}\left(t, \bar{X}(t), \bar{X}_{a}(t), \bar{X}_{\delta}(t), u, \bar{P}(t), \bar{P}^{a}(t), H_{\bar{P}}(t), H_{\bar{P}^{a}}(t)\right) .
\end{aligned}
$$

Note that the adjoint equations derived here are different from those defined in [13]: instead of the adjoint equations for a triple of stochastic processes in [13], we have those for paired stochastic processes. In addition, instead of a classic controlled BSDE as in [13], one of the adjoint equations here is described by an anticipated BSDE. Note also that the Hamiltonian and adjoint equations here are both different from those defined in [14].

Similarly, we can generalize Theorem 4.2 to obtain the following sufficient stochastic maximum principle for the control problem (56). In particular, it requires weaker assumptions than those in [13, Theorem 2.2] and in [14, Theorem 3.1], of which our result is therefore a generalization.

Theorem 5.3. In addition to modified Hypotheses I \& II, we assume further that the functions $b, \sigma$ and $G$ are continuously differentiable with respect to $(x, y, z)$, that $g$ is continuously differentiable with respect to $(x, y)$ and that $\mathcal{H}_{a}\left(t, x, y, z, u, p, r, h_{p}, h_{r}\right)$ is concave with respect to $(x, y, z, u)$. Let $\bar{u} \in \mathcal{U}, \bar{X}$ be the solution to the controlled SDDE (55) associated with $\bar{u}$, and $\left(\bar{P}, H_{\bar{P}}\right)$ and $\left(\bar{P}^{a}, H_{\bar{P}^{a}}\right)$ be the solutions to the adjoint equations (5.2) and (5.2) associated with $(\bar{u}, \bar{X})$. If $\left(\bar{u}, \bar{X}, \bar{P}, \bar{P}^{a}\right)$ satisfies (60), then $\bar{u}$ is an optimal solution for the control problem (56).

We note that, if (55) is independent of $X_{a}$, then the Hamiltonian and the associated adjoint equations involved in the maximum principles for the control problem (56) coincide with those obtained in Section 4 for the corresponding control problem with just discrete delay. Hence, our results in Section 4 become a special case of those for the optimal control problems with both discrete and exponential moving average delays. 
Finally, we finish the paper by considering the following simple control problem with both discrete and exponential moving average delays. Note that it usually cannot be solved using the results either of [14] or of [13] as, for the former, $g$ needs to be independent of $y$ and, for the latter, the parameters need to satisfy the constraints

$$
f_{3} e^{-\lambda \delta}=b_{1}(t) a_{3}, \quad b_{1}(t) \neq 0 \quad \text { and } \quad \frac{e^{-\lambda \delta} f_{1}(t)}{b_{1}(t)}-\lambda=a_{1}(t)+b_{1}(t) e^{\lambda \delta},
$$

to ensure that one of the adjoint processes there be identically zero.

Example 5.1. As in Example 4.1, we set $n=m=1$. Suppose that $\mathbb{U}=\mathbb{R}$; that

$$
\begin{aligned}
b(t, x, y, z, u) & =a_{1}(t) x+f_{1}(t) y+b_{1}(t) z+c_{1}(t) u \\
\sigma(t, x, y, z, u) & =a_{2}(t) x+f_{2}(t) y+b_{2}(t) z+c_{2}(t) u
\end{aligned}
$$

and that

$$
G\left(t, x, y, x_{d}, u\right)=\frac{1}{2} c_{3}(t) u^{2} \quad \text { and } \quad g(x, y)=a_{3} x+f_{3} y,
$$

where $a_{1}, a_{2}, a_{3}, b_{1}, b_{2}, c_{1}, c_{2}, c_{3}$ are as given in Example 4.1, $f_{1}$ and $f_{2}$ are $\mathbb{R}$-valued continuous functions and $f_{3} \in \mathbb{R}$ is a constant.

Similarly to Example 4.1, it can be verified that this control problem can be reformulated as a particular convex problem, where the corresponding Assumptions I, II \& III are satisfied. The Hamiltonian for this problem is given by

$$
\begin{aligned}
\mathcal{H}_{a}\left(t, x, y, z, u, p, r, h_{p}, h_{r}\right)= & \left\{a_{1}(t) x+f_{1}(t) y+b_{1}(t) z+c_{1}(t) u\right\} p \\
& +\left\{a_{2}(t) x+f_{2}(t) y+b_{2}(t) z+c_{2}(t) u\right\} h_{p} \\
& +\left\{x-\lambda y-e^{-\lambda \delta} z\right\} r-\frac{1}{2} c_{3}(t) u^{2},
\end{aligned}
$$

which satisfies the concavity condition required by Theorem 5.3. The associated paired adjoint processes are

$$
\left\{\begin{aligned}
d P(t)=- & \left\{a_{1}(t) P(t)+P^{a}(t)+a_{2}(t) H_{P}(t)\right. \\
& +E\left[\left\{b_{1}(t) P(t+\delta)-e^{-\lambda \delta} P^{a}(t+\delta)\right.\right. \\
& \left.\left.\left.\quad+b_{2}(t) H_{P}(t+\delta)\right\} I_{[0, T-\delta]}(t) \mid \mathcal{F}(t)\right]\right\} d t \\
& +H_{P}(t) d B(t), \quad t \in[0, T] \\
P(T)=-a_{3} &
\end{aligned}\right.
$$


and

$$
\left\{\begin{aligned}
d P^{a}(t)= & -\left\{f_{1}(t) P(t)-\lambda P^{a}(t)+f_{2}(t) H_{P}(t)\right\} d t \\
& +H_{P^{a}}(t) d B(t), \quad t \in[0, T], \\
P^{a}(T)= & -f_{3} .
\end{aligned}\right.
$$

By taking the derivative, with respect to $u$, of $\mathcal{H}_{a}$, we find that

$$
\bar{u}(t)=\frac{1}{c_{3}(t)}\left\{c_{1}(t) \bar{P}(t)+c_{2}(t) H_{\bar{P}}(t)\right\}
$$

is an optimal control for the problem, where $\left(\bar{P}, H_{\bar{P}}\right)$, together with $\left(\bar{P}^{a}, H_{\bar{P}^{a}}\right)$, is the solution of the paired adjoint equations. It can be verified that the pair of adjoint equations in this example admits a unique solution. In particular, since $P(T)$ and $P^{a}(T)$ are both constants, $H_{P}(t)=H_{P^{a}}(t) \equiv 0$. Hence, this delayed control problem has a deterministic solution.

\section{References}

[1] Bismut, J. M. (1973). Conjugate convex functions in optimal stochastic control. J. Math. Anal. Appl. 44, 384-404.

[2] Bismut, J. M. (1978). An introductory approach to duality in optimal stochastic control. Society for Industrial and Applied Mathematics Review 20, 62-78.

[3] Chang, M. H., PAng, T. And YAng, Y. (2011). A stochastic portfolio optimization model with bounded memory. Math. Operat. Res. 36, 604-619.

[4] Chen, L. And Wu, Z. (2010). Maximum principle for the stochastic optimal control problem with delay and application. Automatica 46, 1074-1080.

[5] Cont, R. And Fournié, D.-A. (2013). Functional Itô calculus and stochstic integral representation of martingales. Ann. Prob. 41, 109-133.

[6] Dupire, B. (2009). Functional Itô calculus. Portfolio Research Paper 2009-04, Bloomberg.

[7] Elsanosi, I., Øksendal, B. and Sulem, A. (2000). Some solvable stochastic control problems with delay. Stochastics and Stochastics Reports 71, 69-89. 
[8] Hobson, D. G. And Rogers, L. C. G. (1998). Complete models with stochastic volatility. Math. Finance 8, 27-48.

[9] Karatzas, I. And Shreve, S. E. (1991). Brownian Motion and Stochastic Calculus, 2nd edn. Springer, New York.

[10] Larssen, B. (2002). Dynamic programming in stochastic control of systems with delay. Stochastics and Stochastics Reports 74, 651-673.

[11] Larssen, B. and Risebro, N. H. (2003). When are HJB-equations for control problems with stochastic delay equations finite dimensional? Stochastic Analysis and Applications 21, 643-671.

[12] Meng, Q. And Shen, Y. (2016). Optimal control for stochastic delay evolution equations. Appl. Math. Optim. 74, 53-89.

[13] Øksendal, B. And Sulem, A. (2001). A maximum principle for optimal control of stochastic systems with delay with applications to finance. In Optimal Control and Partial Differential Equations-Innovations and Applications, eds. J. L. Menaldi, E. Rofman and A. Sulem, IOS Press, Amsterdam, pp. 64-79.

[14] Øksendal, B., Sulem, A. and Zhang, T. (2011). Optimal control of stochastic delay equations and time-advanced backward stochastic differential equations. Adv. Appl. Prob. 43, 572-596.

[15] Peng, S. And Yang, Z. (2009). Anticipated backward stochastic differential equations. Ann. Prob. 37, 877-902.

[16] Rockafellar, R. T. (1968). Integrals which are convex functionals. Pacific Journal of Mathematics 24, 525-539.

[17] Rockafellar, R. T. (1969). Convex Analysis. Princeton University Press, New Jersey.

[18] Rockafellar, R. T. (1970). Conjugate convex functions in optimal control and the calculus of variations. J. Math. Anal. Appl. 32, 174-222.

[19] Rockafellar, R. T. (1974). Conjugate Duality and Optimization. Society for Industrial and Applied Mathematics, Philadelphia. 
[20] Tsoutsinos, G. I. And Vinter, R. B. (1995). Duality theorems for convex problems with time delay. J. Optimization Theory Appl. 87, 167-195.

[21] Yong, Z. And Zhou, X. (1999). Stochastic Controls: Hamiltonian Systems and HJB Equations. Springer, New York. 